\title{
Some Aspects of the Hydrodynamics of the Microencapsulation Route to NIF Mandrels
}

\author{
P. M. Gresho
}

This paper was prepared for submittal to the

Target Fabrication Meeting '98

Jackson Hole, WY

April 19-23, 1998

October 20, 1998

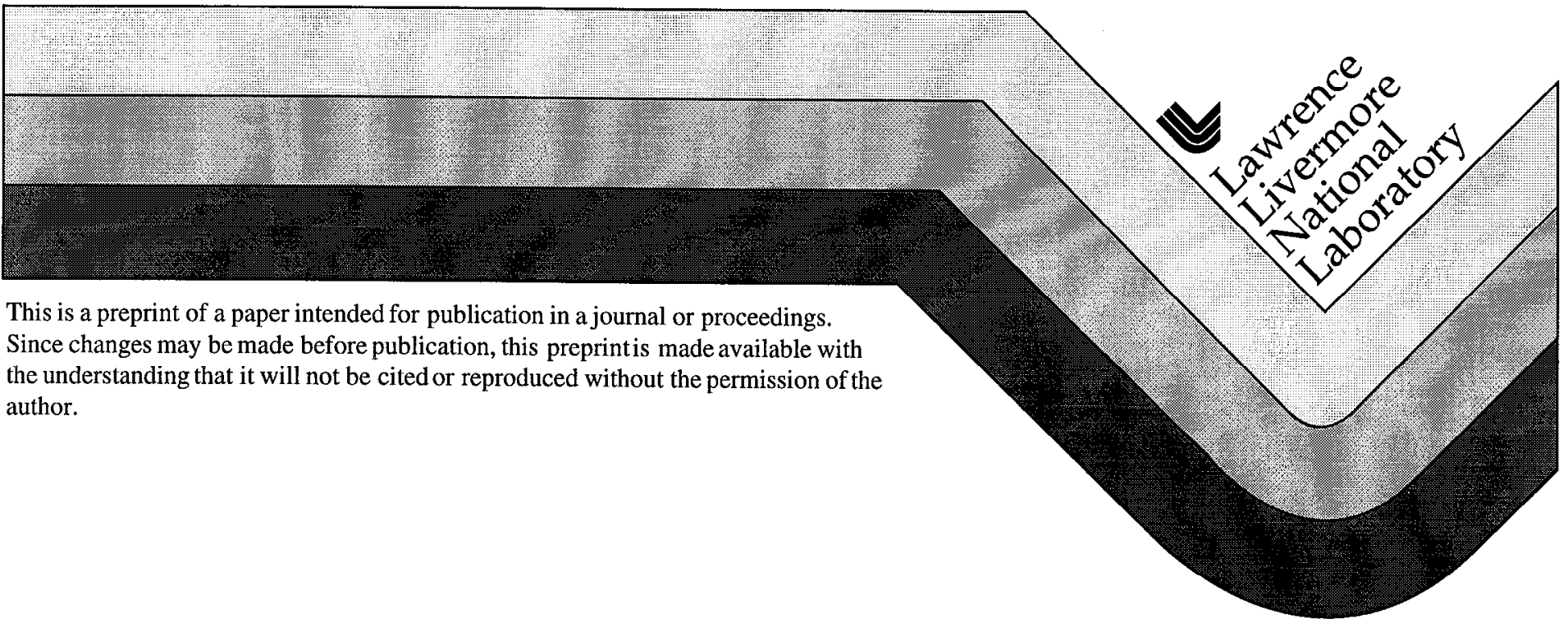




\section{DISCLAIMER}

This document was prepared as an account of work sponsored by an agency of the United States Government. Neither the United States Government nor the University of California nor any of their employees, makes any warranty, express or implied, or assumes any legal liability or responsibility for the accuracy, completeness, or usefulness of any information, apparatus, product, or process disclosed, or represents that its use would not infringe privately owned rights. Reference herein to any specific commercial product, process, or service by trade name, trademark, manufacturer, or otherwise, does not necessarily constitute or imply its endorsement, recommendation, or favoring by the United States Government or the University of California. The views and opinions of authors expressed herein do not necessarily state or reflect those of the United States Government or the University of California, and shall not be used for advertising or product endorsement purposes. 
Some Aspects of the Hydrodynamics of the Microencapsulation Route to NIF Mandrels

\section{P.M. Gresho}

Lawrence Livermore National Laboratory 
Abstract

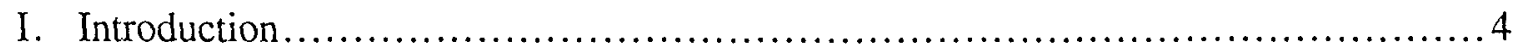

II. Hydrostatics - the Young-Laplace Eqn. .......................................... 7

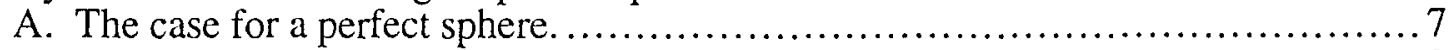

B. A simple droplet in a density column........................................ 8

C. A compound / encapsulated droplet ........................................ 11

III. Steady Hydrodynamics......................................................... 12

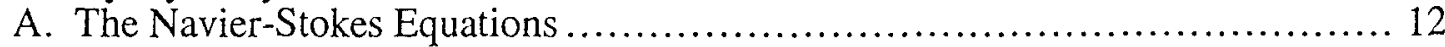

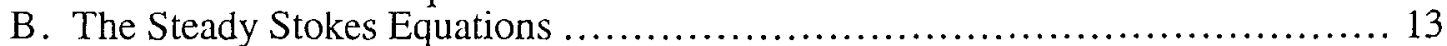

1. Drag force ........................................................... 13

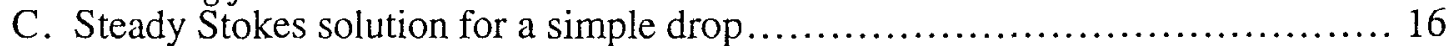

1. No-slip no longer applies ............................................ 16

2. Drag force and terminal speed ....................................... 18

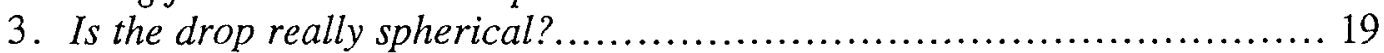

4. Non-constant surface tension: Marangoni flow ......................... 20

D. Steady Stokes Flow for a Compound/Encapsulated Drop ..................... 21

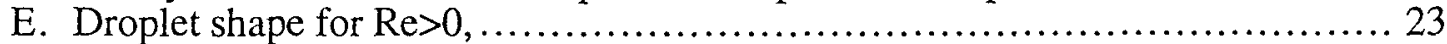

1. Simple drops in free-fall .......................................... 23

2. Simple drop in a linear shear flow ................................... 24

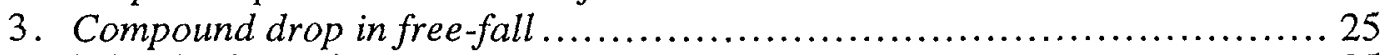

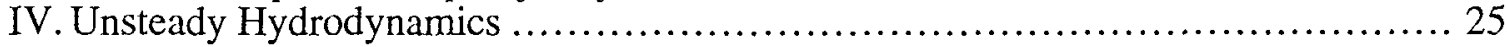

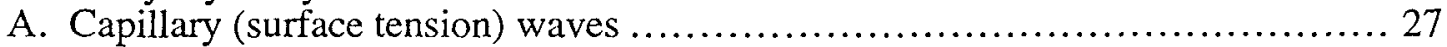

1. Ideal waves, simple and compound drops ............................. 27

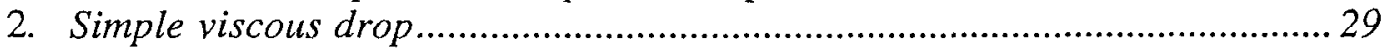

3. Compound viscous drop ........................................... 30

B. Time-dependent potential flow, concentric spheres ........................... 30

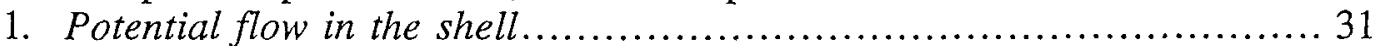

2. Potential flow in the core and the host .................................. 32

C. The solid sphere ........................................................ 34

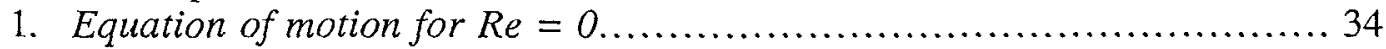

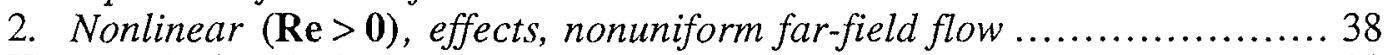

D. The simple drop in Stokes flow............................................. 38

E. The compound drop in Stokes flow ...................................... 40

1. Equations of motion................................................ 40

2. Core centering .......................................................... 45

V. Applications................................................................ 47

A. Static droplet in a density column.......................................... 48

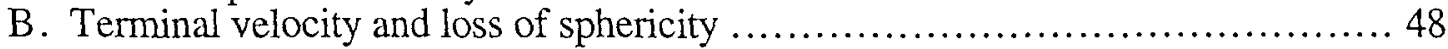

1. Stokes flow ........................................................ 48

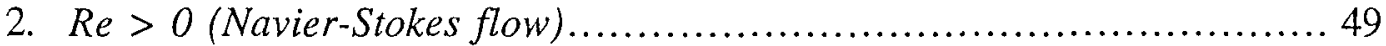

3. Droplet shape .................................................... 49

C. Droplet shape in a linear shear flow ....................................... 50

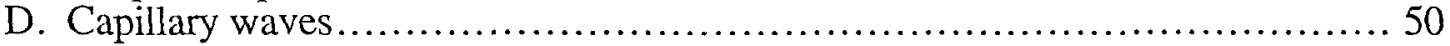

1. Inviscid simple drop................................................ 50

2. Viscous simple drop.............................................. 50

3. Inviscid compound drop ............................................. 51

E. Initial accelerations ...................................................... 51

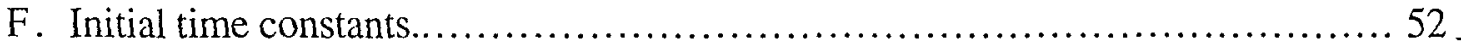

IV. Conclusions and Future Work.............................................. 54

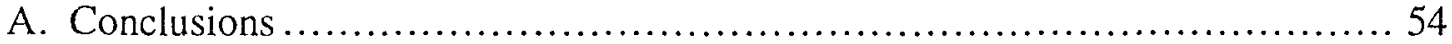




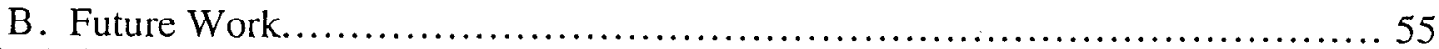

VII. Acknowledgements ...................................................... 56

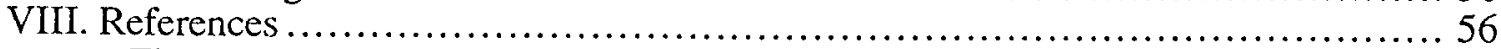

Fig. 1 Compound drop............................................... 60

Fig. 2 Simple Drop; Steady Streamlines $\left(\mu_{i}=\mu_{o}\right) \ldots \ldots \ldots \ldots \ldots \ldots \ldots \ldots \ldots \ldots \ldots$

Fig. 3 Streamlines for a Compound Droplet..................................62 62

Fig. 4 Rising/Falling Compound Droplets — at $U_{T}, \ldots \ldots \ldots \ldots \ldots \ldots \ldots \ldots \ldots$

Fig. 5 First two modes for a compound drop.............................. 64

Fig. 6 Streamlines and Isobars for an (upward) accelerating shell...............66 65

Fig. 7 Stokes' Law Correction Factor...................................... 66 


\begin{abstract}
Spherical plastic shells for use as mandrels for the fabrication of ICF (Inertial Confinement Fusion) target capsules can be produced by solution-based microencapsulation techniques. The specifications for these mandrels in terms of sphericity are extremely rigorous, and it is clear that various aspects of the solution hydrodynamics associated with their production are important in controlling the quality of the final product. This paper explores what we know (and need to know) about the hydrodynamics of the microencapsulation process in order to lay the foundation for process improvements as well as identify inherent limits.
\end{abstract}

\title{
I. Introduction
}

Target capsules for use at the National Ignition Facility (NIF), beginning in about 2005, will be about $2 \mathrm{~mm}$ in diameter with 150 to $200 \mu \mathrm{m}$ walls composed of plastic or beryllium. Although target designs (see for example Haan, 1995, Dittrich, 1997, or other papers in this issue of Fusion Technology) are still evolving, it is clear that capsulesphericity and surface finish will be critically important. The current surface specifications are presented elsewhere in this volume (Cook et.al., 1998), but it is sufficient to say at this point that surface finish and sphericity at least as good as what is currently available with $0.5 \mathrm{~mm}$ diameter Nova-scale capsules will be required, even though the NIF capsules are four times as large.

Most capsule fabrication scenarios involve producing an initial spherical shell (or bead) of poly( $\alpha$-methylstyrene) (P $\alpha M S)$, which is used as the template for the final capsule - using methods that need not concern us here. (See numerous papers in this issue of Fusion Technology). We will focus exclusively upon the processes that are used to produce this initial template shell (or bead). PoMS shells can be produced by microencapsulation techniques in which a triple orifice droplet generator produces water droplets surrounded by a layer of a non-aqueous solution of PaMS; these compound droplets are suspended in an aqueous bath. The microencapsulated droplet can be described (see Figure 1) in terms of the radius of the core water droplet and compound droplet, $R_{C}$ and $R_{S}$, respectively. Also shown in Figure 1 is the coordinate frame we will use. A significant portion of the paper will deal with the simpler case of a simple droplet (no core) or a solid sphere suspended in an aqueous bath, and for these discussions we will. 
simply label relevant variables with $i$ and $o$ to represent the droplet (inside) and host fluid (outside). The radius of such droplets will simply be given by $R$.

With gentle heating of the bath, the organic solvent of the non-aqueous "oil" shell dissipates (diffuses) into the aqueous bath (host) leaving a solid polymer shell with an aqueous core that can later be air dried to remove the 'water'. Bead variants on this approach avoid the aqueous core (Czechowicz and Stephens, 1998) or directly attempt to sphericalize solid PaMS beads through a melt phase (Letts et al., 1998). The key to success in all of these approaches is to minimize perturbations to the shell or bead sphericity. In most of this paper we will focus on only the lowest order capsule deformations, and we have as our target "mode 2 " deformations of less than $1 \mu \mathrm{m}$. The need to meet such stringent tolerances has led to the need (or at least the desire) to understand the fluid dynamics involved in the fabrication of these shells (or beads) at a very basic level, with the hope that such understanding would contribute sufficient additional knowledge to the experimental studies so that process improvements could be developed.

Among the hydrodynamic issues that are relevant to this process are the following: (1) Surface tension (our good friend) tries to make droplets attain a spherical shape, (2) droplet motion tends to deform the droplets away from spherical, (3) density mismatch between core and shell (always present to some extent, although partially controllable) tends to cause de-centering of the core (which can also contribute to loss of sphericity) and, at its worst, a loss of shell owing to bursting, (4) non-constant surface tension will cause additional fluid flow that could also cause loss of sphericity, and (5) the (desirable) physics of 'core-centering', seemingly caused by dynamic perturbations at the two fluid interfaces (such as capillary wave generation or externally-imposed acoustic oscillations) needs to be better-understood. 
To address these and related issues, we have explored the following fundamental fluid dynamic phenomena: (1) The shape of static droplets when density variations tend to distort the otherwise-spheres, (2) the terminal (settling) velocity of compound droplets and their stability (core remains in shell?), (3) the rate of attainment of terminal velocity, (4) the special 'physics' of thin-shelled droplets (nearly our case since $R_{c} / R_{s} \cong 0.9$ ), some of which is related to potential flows ( $\mathrm{Re}=\infty$ : $\mathrm{Re}=$ Reynolds number) and some of which is at the other extreme: Stokes flow $(\operatorname{Re}=0)$, (5) the effects of nonlinearity (the advection term in the governing Navier-Stokes equations) on droplet sphericity, (6) the temporal response and damping rate of droplets that are perturbed from sphericity-via capillary wave analysis, (7) the general equations of motion of core and shell, and finally, (8) some discussion of the core-centering phenomena.

Some of the above are treated in a fair amount of detail, including simpler related phenomena (often with complete derivations) as a sort of 'tutorial', because another objective of this paper is to introduce a few perhaps-esoteric fluid-dynamical phenomena to that portion of the physics community that is interested in this particular method of target fabrication. Others on the list will be treated only briefly, sometimes owing to their extreme complexity (we then send the reader to the original research papers), and other times because we still don't know how to do a better job. One of the problems encountered during the research phase, which unfortunately happens in any developing field of science, is that the history of the detailed analysis and understanding of the dynamics of particles, drops, and bubbles is somewhat 'polluted' with spurious results ... most of which eventually become corrected.

We somewhat apologetically (to some) must point out that much of (theoretical) fluid mechanics is one or another 'exercise in applied mathmatics'-which may well blur the picture for some readers. Partly for non-mathematically-oriented readers (mathematicallychallenged?), we present all of the 'theory' in the bulk of the paper and save applications of the theory to the last section. This may be useful to those who are more interested in the 'bottom line' than the details of how we got there. If this turns out to be the majority, please except our apologies for too much deflectionary detail. 


\section{Hydrostatics-the Young-Laplace Eqn.}

\section{A. The case for a perfect sphere.}

We begin 'statically', $\boldsymbol{u}=0$, for simplicity. The static shape of 'free' fluid surfaces is governed largely by the so-called Young-Laplace eqn. (sometimes called Laplaces' formula), which applies pointwise at a stationary interface:

$$
\Delta \mathrm{P}=2 \gamma H=\gamma\left(k_{1}+k_{2}\right)=\gamma\left(\frac{1}{R_{1}}+\frac{1}{R_{2}}\right)=\gamma \nabla \cdot n=\mathcal{N}_{s} \cdot n,
$$

where $\Delta P$ is the pressure jump across the interface, $\gamma$ is the interfacial (surface) tension, $\mathrm{H}$ is the mean curvature, $k_{1}$ and $k_{2}$ are the principal curvatures, $R_{1}$ and $R_{2}$ are the two principal radii of curvature, $\boldsymbol{n}$ is the unit normal vector, and $\nabla_{s}$ is the (2-dimensional) surface gradient. $\left(\nabla \cdot \boldsymbol{n}=\nabla_{s} \cdot \boldsymbol{n}\right.$ because :

$$
\nabla_{s} \cdot \boldsymbol{n} \equiv[(\underline{\underline{I}}-\boldsymbol{n n}) \cdot \nabla] \cdot \boldsymbol{n}=(\nabla-\boldsymbol{n} \boldsymbol{n} \cdot \nabla) \cdot \boldsymbol{n}=\nabla \cdot \boldsymbol{n}-\boldsymbol{n}(\boldsymbol{n} \cdot \nabla) \cdot \boldsymbol{n}=\nabla \cdot \boldsymbol{n}-n_{i} n_{j} \partial n_{i} / \partial x_{j}=\nabla \cdot \boldsymbol{n}
$$

$-\frac{1}{2} n_{j} \partial n_{i}^{2} / \partial x_{j}=\nabla \cdot n$ because $n_{i}^{2}=1$ and thus $\left.\partial n_{i}^{2} / \partial x_{j}=0\right)$. In a gravitational field with constant density fluids, $\Delta P$ is replaced by $\Delta P \pm \Delta \rho g z$ and the static condition can then only exist if appropriate "boundary conditions" (BC's) permit it-or if $2 \gamma H$ is very large relative to the hydrostatic pressures. An appeal to authority seems appropriate at this point (Batchelor 1967): "A case in which the equilibrium shape of the interface is obvious is that of a mass of one fluid immersed in a second fluid, e.g. a mist droplet in air or a gas bubble in water. Provided that either the volume of the drop or bubble or the difference between the densities of the two sides of the interface is sufficiently small, we may ignore the effect of gravity. The pressure is then uniform in each fluid and the pressure jump in (1) is constant over the interface. An unbounded surface with a constant sum of principal curvatures is spherical, and this must be the equilibrium shape of the surface. This result also follows from the fact that in a state of (stable) equilibrium the energy of the surface must be a minimum consistent with a given value of the volume of the drop or bubble, and a sphere is the shape which has least surface area for given volume." For a sphere, (1) of course simplifies to

$$
\Delta P=2 \gamma / R
$$

which relates the outside to inside pressure (both constant) for a (simple) spherical drop of radius $R ; P_{\text {inside }}=P_{\text {outside }}+2 \gamma / R>P_{\text {outside }}$ because $\gamma$ acts like a skin under tension. 


\section{B. A simple droplet in a density column}

This is a situation in which a droplet could remain motionless even though $\Delta \rho \neq 0$; i.e. a constant density droplet $\left(\rho_{0}\right)$ in a (linearly) stably - stratified "host" fluid, with density

$$
\rho(z)=\rho_{0}(1-\beta z)
$$

where $\beta \ll 1 / R$ and the origin of the (vertical, upward-pointing) coordinate system was taken to be at the droplet's equilibrium position, will no longer be spherical. The YoungLaplace equation can be used to obtain, analytically, the droplet's shape on the assumption (valid for most cases of interest to us) that its shape is very close to spherical. This is done as follows (with special thanks to Howard Stone):

(i) The hydrostatic pressure in the drop is (via $\partial P / \partial z=-\rho g$ )

$$
P_{i}(z)=\bar{P}_{1}-\rho_{0} g z
$$

and that outside the drop is

$$
P_{0}(z)=\bar{P}_{2}-\rho_{0} g z+\frac{1}{2} \rho_{0} g \beta z^{2}
$$

where $\bar{P}_{1}$ and $\bar{P}_{2}$ are constants.

(ii) The Young-Laplace equation now gives

$$
\gamma \nabla \cdot \boldsymbol{n}=\left(\bar{P}_{1}-\bar{P}_{2}\right)-\frac{1}{2} \rho_{0} g \beta z^{2},
$$

where $z$ is now on the drop surface.

(iii) The curvature can be obtained via the so-called "method of domain perturbations" (e.g. Leal 1992) by assuming an axisymmetric droplet shape of the form $r(\theta)=R[1+\varepsilon f(\theta)]$, or

$$
F(r, \theta)=0 \equiv r-R[1+\varepsilon f(\theta)]
$$

where $\mathrm{R}$ is the nominal (and constant) drop radius and $\varepsilon<<1$. The unit normal vector at $r(\theta)$ is then

$$
n=\nabla F /|\nabla F|
$$


where

$$
\begin{aligned}
\nabla F & =e_{r} \frac{\partial F}{\partial r}+\frac{1}{r} e_{\theta} \frac{\partial F}{\partial \theta} \\
& =e_{r}-e_{\theta} \frac{\varepsilon R}{r} f^{\prime}(\theta)
\end{aligned}
$$

where $e_{r}, e_{\theta}$ are the unit vectors, in (axi-symmetric) spherical coordinates, in the $r$ - and $\theta$ - directions, respectively.

Thus,

$$
\begin{aligned}
|\mathrm{V} F|^{2} & =1+\left[\varepsilon R f^{\prime}(\theta) / r\right]^{2} \text { and } \\
n & =\frac{e_{r}-e_{\theta} \frac{\varepsilon R}{r} f^{\prime}(t)}{\sqrt{1+\left(\varepsilon R f^{\prime} / r\right)^{2}}} \equiv e_{r} n_{r}+e_{\theta} n_{\theta} .
\end{aligned}
$$

Finally,

$$
\nabla \cdot n=\frac{1}{r^{2}} \frac{\partial}{\partial r}\left(r^{2} n_{r}\right)+\frac{1}{r \sin \theta} \frac{\partial}{\partial \theta}\left(n_{\theta} \sin \theta\right)
$$

and we will treat-and approximate-each term separately.

$$
\begin{aligned}
\frac{\partial}{\partial r}\left(r^{2} n_{r}\right) & =\frac{\partial}{\partial r}\left[\frac{r^{2}}{\sqrt{1+\left(\varepsilon R f^{\prime} / r\right)^{2}}}\right] \\
& =\frac{\partial}{\partial r}\left\{r^{2}\left[1-\frac{1}{2}\left(\varepsilon R f^{\prime} / r\right)^{2}\right]\right\}+O\left(\varepsilon^{3}\right) \cong 2 r
\end{aligned}
$$

$$
\begin{aligned}
\frac{\partial}{\partial \theta}\left(n_{\theta} \sin \theta\right) & =-\frac{\varepsilon R}{r} \frac{\partial}{\partial \theta}\left[\frac{f^{\prime}(\theta) \sin \theta}{\sqrt{1+\left(\varepsilon R f^{\prime} / r\right)^{2}}}\right] \\
& \cong-\frac{\varepsilon R}{r} \frac{\partial}{\partial \theta}\left\{f^{\prime}(\theta) \sin \theta\left[1-\frac{1}{2}\left(\varepsilon R f^{\prime} / r\right)^{2}\right]\right\} \\
& \cong-\frac{\varepsilon R}{r}\left\{f^{\prime}(\theta) \cos \theta+f^{\prime \prime}(\theta) \sin \theta+O\left(\varepsilon^{2}\right)\right\}
\end{aligned}
$$

Thus, to $O\left(\varepsilon^{2}\right)$, we have, using $\frac{1}{r} \cong \frac{1}{R}(1-\varepsilon f)$, 


$$
\nabla \cdot \boldsymbol{n}=\frac{1}{R}\left\{2-\varepsilon\left[2 f+\frac{\cos \theta}{\sin \theta} f^{\prime}(\theta)+f^{\prime \prime}(\theta)\right]\right\} .
$$

(iv) Next, (6) gives, using $z \cong R \cos \theta$ on the surface, again to $O\left(\varepsilon^{2}\right)$,

$$
\begin{aligned}
& \frac{\gamma}{R}\left\{2-\varepsilon\left[2 f+f^{\prime} \frac{\cos \theta}{\sin \theta}+f^{\prime \prime}\right]\right\}=\bar{P}_{1}-\bar{P}_{2}-\frac{1}{2} \rho_{0} g \beta R^{2} \cos ^{2} \theta, \text { or } \\
& \varepsilon\left[f^{\prime \prime}(\theta)+\frac{\cos \theta}{\sin \theta} f^{\prime}(\theta)+2 f(\theta)\right]=2-\frac{R\left(\bar{P}_{1}-\bar{P}_{2}\right)}{\gamma}+\frac{\rho_{0} g \beta R^{3}}{2 \gamma} \cos ^{2} \theta
\end{aligned}
$$

Finally, since $\beta \ll 1$, we can identify $\varepsilon$ with $\beta$ via

$$
\varepsilon \equiv \frac{\rho_{0} g \beta R^{3}}{2 \gamma},
$$

a (stratification) Bond number, to give, finally,

$$
\begin{aligned}
f^{\prime \prime}(\theta)+\frac{\cos \theta}{\sin \theta} f^{\prime}(\theta)+2 f(\theta) & =\cos ^{2} \theta+\frac{1}{\varepsilon}\left[2-\frac{R\left(\bar{P}_{1}-\bar{P}_{2}\right)}{\gamma}\right]+O(\varepsilon) \\
& \equiv \cos ^{2} \theta+c+O(\varepsilon)
\end{aligned}
$$

as the governing equation-along with (7) - for the surface shape,

(v) The solution to this linear ODE that satisfies $f^{\prime}(0)=f^{\prime}(\pi / 2)=0$

is, to $O(\varepsilon)$,

$$
f(\theta)=c / 2+\frac{1}{4} \sin ^{2} \theta
$$

(vi) In order to conserve volume to $O(\varepsilon)$, it is required that

$$
\int_{0}^{\pi} f(\theta) \sin \theta d \theta=0
$$

which requires that $c=-1 / 6$ to give

$$
\bar{P}_{1}-\bar{P}_{2}=\frac{2 \gamma}{R}(1+\varepsilon / 6)+O\left(\varepsilon^{2}\right) ;[\text { cf. (2)] and }
$$


the final solution is

$$
\begin{aligned}
f(\theta) & =-\frac{1}{12}+\frac{1}{4} \sin ^{2} \theta \\
& =-\frac{1}{12}\left[3 \cos ^{2} \theta-1\right] \\
& =-\frac{1}{6} P_{2}(\cos \theta)
\end{aligned}
$$

where $P_{2}$ is the second-order Legendre polynominal ("mode 2").

Remark: For small $\varepsilon, P_{2}(\cos \theta)$ gives a droplet shape that is very close to an oblate cllipsoid (fatter at the equator, thinner at the poles-like Mother Earth).

(vi) Finally, the droplet shape (the object of the exercise) is given by

$$
r(\theta)=R\left[1-\frac{\rho_{0} g \beta R^{3}}{12 \gamma} \cdot P_{2}(\cos \theta)\right]
$$

giving a MooR (Maximum-out-of-Round) of

$$
M o o R \equiv 2[r(\pi / 2)-r(0)]=\rho_{0} g \beta R^{4} / 4 \gamma=\varepsilon / 2
$$

which is accurate to $O\left(\varepsilon^{2}\right)$ and, of course, is only valid if $\varepsilon<<1$.

To conclude this section, we note that a static configuration requires a constant surface tension-which may not always be the case when thermal or concentration gradients are present--the latter possibly occurring if surfactants are present. We shall return to this "Marangoni effect" in a later section after we permit fluid motion.

\section{A compound / encapsulated droplet}

For this case, we can only have a truly static situation if the densities of the three liquids are all the same. The droplet will then still be spherical, with (2) applying in both core and shell ; i.e., we then have, in the absence of gravity (or the 'dominance' of surface tension)

$$
P_{\text {shell }}=P_{\text {host }}+2 \gamma_{s} / R_{s}
$$

and 


$$
\begin{aligned}
P_{\text {core }} & =P_{\text {shell }}+2 \gamma_{c} / R_{c} \\
& =P_{\text {hess }}+2 \gamma_{c} / R_{c}+2 \gamma_{s} / R_{s} .
\end{aligned}
$$

The core pressure could be "large" for small droplets. Note that (21) and (22) do not require concentric core and shell.

If gravity is present, the pressures are no longer constant because $P_{\text {host }}$ is no longer constant; it is

$$
P_{\text {host }}=P_{0}-\rho g z
$$

where $P_{0}$ is an arbitrary constant. Again, core centering is not required-although we clearly must always have (for complete encapsulation), $d<R_{s}-R_{c}$, where $\mathrm{d}$ is the distance between centers.

A compound droplet in a density column (linearly stably-stratified, as bcfore, for simplicity) can only be in static equilibrium if $\rho_{c}=\rho_{s}$ and, using (3), $\rho_{s}=\rho_{0}$, where now the origin of $\mathrm{z}$ is at the shell's center-that of the core being irrelevant. In this case, the Young-Laplace equation can again be solved approximately to give $(i)$ a shell shape given by (19), and (ii) a core that is spherical - a pair of results that is, unfortunately, in the opposite sense to that desired for target fabrication. We want a spherical shell, the core sphericity mattering less; c'est la vie--and this brings us to the end of hydrostatics.

\section{Steady Hydrodynamics}

\section{A. The Navier-Stokes Equations}

Since virtually all of the equations to follow are some sort of simplification of the incompressible Navier-Stokes equations (see Gresho and Sani 1998 for details regarding these equations), we begin by stating them-the conservations 'laws' for momentum and mass, respectively:

$$
u \cdot \nabla u+\nabla(P / \rho)=v \nabla^{2} u+f
$$

and

$$
\nabla \cdot \boldsymbol{u}=0
$$

where $\boldsymbol{u}$ is the velocity and $P$ the pressure. The vector $f$ is a body 'force' (e.g. gravity, $g), v \equiv \mu / \rho$ is the (constant) kinematic viscosity, $\mu$ (also constant) is the viscosity and $\rho$ is the constant density. If we have a 'slightly' variable fluid density in a gravity field, ' 
owing to thermal or concentration inhomogenieties, the body force term becomes $f=\frac{\Delta \rho(\boldsymbol{x}, t)}{\rho_{0}} \mathrm{~g}$ with $\rho_{0}$ still constant, and $\Delta \rho \ll \rho_{0}$ and obtained by simultaneously solving the conservation equation for temperature (energy) and / or species concentration-a situation deemed too complex for out present purposes.

In the sequel, we shall also usually assume that the Reynolds number,

$$
\mathrm{Re} \equiv U_{c} R / v
$$

where $U_{c}$ is a characteristic velocity of the flow, is very small- $\operatorname{Re} \ll 1$-which justifies the neglect of the nonlinear inertial/advection term, $\boldsymbol{u} \cdot \nabla \boldsymbol{u}$, from (24). The result is the Stokes equations, which are linear and subject to some analytical solutions for special cases-considered next.

\section{B. The Steady Stokes Equations}

The simplest situation of interest here in which a nonzero velocity is present is described by

$$
\nabla P=\mu \nabla^{2} u+\rho g
$$

and (25), and the plan is to show (in summary form) how these can be used to find the drag force and the terminal velocity, $\boldsymbol{u}_{T}=u_{T} \boldsymbol{e}_{z}$, for a freely falling sphere-first for a rigid (solid) particle, then (in the next two sections) for a simple droplet, and finally for a compound droplet, the latter two of which also require proving that the moving drops actually remain spherical.

\section{Dragforce}

For a sphere translating at a fixed velocity through a quiescent unbounded fluid-or, what is the same and somewhat simpler to deal with initially - the steady flow past a fixed sphere in an unbounded fluid, the Stokes equations for axisymmetric flow become, in spherical coordinates,

$$
\frac{\mu}{r^{2}}\left[\frac{\partial}{\partial r}\left(r^{2} \frac{\partial u_{r}}{\partial_{r}}\right)+\frac{1}{\sin \theta}\left(\sin \theta \frac{\partial u_{r}}{\partial \theta}\right)-2 u_{r}-2 \frac{\partial u_{\theta}}{\partial \theta}-2 u_{\theta} \cot \theta\right]-\frac{\partial P}{\partial r}=\rho g \cos \theta,
$$




$$
\frac{\mu}{r^{2}}\left[\frac{\partial}{\partial r}\left(r^{2} \frac{\partial u_{\theta}}{\partial r}\right)+\frac{1}{\sin \theta} \frac{\partial}{\partial \theta}\left(\sin \theta \frac{\partial u_{\theta}}{\partial \theta}\right)+2 \frac{\partial u_{r}}{\partial \theta}-\frac{u_{\theta}}{\sin ^{2} \theta}\right]-\frac{1}{r} \frac{\partial P}{\partial \theta}=\rho g \sin \theta
$$

and

$$
\frac{1}{r^{2}} \frac{\partial}{\partial r}\left(r^{2} u_{r}\right)+\frac{1}{r \sin \theta} \frac{\partial}{\partial \theta}\left(u_{\theta} \sin \theta\right)=0
$$

and the $\mathrm{BC}$ 's are

$$
\begin{gathered}
u_{r}=u_{\theta}=0 \text { at } r=R \text { (on the sphere) } \\
u_{r}=u_{\infty} \cos \theta \text { and } u_{\theta}=-u_{\infty} \sin \theta \text { at } r \rightarrow \infty,
\end{gathered}
$$

where $u_{\infty}$ is the unidirectional velocity far from the sphere. The solution, first found by Stokes himself in 1851, can be obtained by 'guessing' the following forms;

$$
\begin{aligned}
& u_{r}=u_{\infty} f(r) \cos \theta, \\
& u_{\theta}=u_{\infty} g(r) \sin \theta,
\end{aligned}
$$

and

$$
P=P_{0}-\rho g r \cos \theta+h(r) \cos \theta \text {, }
$$

from which (28) yields a coupled set of ODE's for $f, g$, and h, which can be combined (see, e.g. Leal 1992 for details) into a single fourth order ODE:

$$
\frac{r^{4}}{8} f^{(i v)}+r^{3} f^{\prime \prime \prime}+r^{2} f^{\prime \prime}-r f^{\prime}=0
$$

which is Euler's differential equation, having simple solutions of the form $f(r)=r^{k}$, where (here) $k=-3,-1,0$, and 2. Upon applying the easily-derived BC's for $f(r)$, the 'final' solution, with $g(r)$ and $h(r)$ following from (28c) and (28b), respectively, is

$$
\begin{gathered}
u_{r}=u_{\infty}\left[1-\frac{3}{2}\left(\frac{R}{r}\right)+\frac{1}{2}\left(\frac{R}{r}\right)^{3}\right] \cos \theta, \\
u_{\theta}=-u_{\infty}\left[1-\frac{3}{4}\left(\frac{R}{r}\right)-\frac{1}{4}\left(\frac{R}{r}\right)^{3}\right] \sin \theta,
\end{gathered}
$$

and

$$
P=P_{0}-\rho g r \cos \theta-\frac{3 \mu u_{\infty}}{2 R}\left(\frac{R}{r}\right)^{2} \cos \theta \text {. }
$$


We are finally ready to compute the drag force, some of which comes from shear stress and the rest of which comes from the pressure. The shear stress is

$$
\tau_{r \theta}=-\mu\left[r \frac{\partial}{\partial r}\left(\frac{u_{\theta}}{r}\right)+\frac{1}{r} \frac{\partial u_{r}}{\partial \theta}\right]
$$

in general, and

$$
\tau_{r \theta}=\frac{3}{2} \frac{\mu u_{\infty}}{R}\left(\frac{R}{r}\right)^{4} \sin \theta
$$

in particular-from (31a) and (31b). The $z$-component of the resulting viscous force (the flow at infinity is in the $\mathrm{z}$-direction) is the viscous friction/drag which, after integrating over the surface of the sphere, is

$$
\begin{aligned}
F_{y} & =2 \pi \int_{0}^{\pi}\left(\left.\tau_{r \theta}\right|_{r=R} \cdot \sin \theta\right) R^{2} \sin \theta d \theta \\
& =4 \pi \mu u_{\infty} .
\end{aligned}
$$

The $z$-component of the pressure force is $-P \cos \theta$ and its contribution to the drag is thus (noting that the normal viscous stress, $\tau_{r r}=2 \mu \partial u_{r} / \partial r=0$ at $r=R$ )

$$
\begin{aligned}
F_{p} & =2 \pi \int_{0}^{\pi}\left(-\left.P\right|_{r=R} \cdot \cos \theta\right) R^{2} \sin \theta d \theta \\
& =\frac{4}{3} \pi R^{3} \rho g+2 \pi \mu R u_{\infty},
\end{aligned}
$$

the former (the buoyancy force of the fluid on the solid) coming from the hydrostatic portion of the pressure $(-\rho g r \cos \theta)$ and the latter from the "viscous" pressure (the constant portion integrates to zero). The sum of $F_{v}$ and $F_{P}$ is the total "drag" felt by the sphere:

$$
F_{D}=\frac{4}{3} \pi R^{3} \rho g+6 \pi \mu R u_{\infty}
$$

the latter term being the famous "Stokes law." Note that $2 / 3$ of the viscous drag comes from the shear stress and $1 / 3$ from the pressure (the so-called "form drag").

2. Terminal Velocity 
It is now a very simple matter to determine the terminal velocity: just equate the upward-directed force by the fluid and given by (35) to the downward force caused by gravity acting on the solid sphere of density $\rho_{s}$; i.e.,

or

$$
\frac{4}{3} \pi R^{3} \rho_{s} g=\frac{4}{3} \pi R^{3} \rho g+6 \pi \mu R u_{\infty}
$$

$$
u_{\infty} \equiv u_{T}^{s}=\frac{2}{9} \frac{g R^{2}}{\mu}\left(\rho-\rho_{s}\right) \text {, }
$$

which is positive for a falling sphere $\left(\rho_{s}>\rho\right)$ and negative for a buoyant one $\left(\rho_{s}<\rho\right)$. [Recall that $u_{\infty}$ is the relative fluid velocity.] Later, we shall see how quickly $u_{T}^{s}$ is approached by solving the transient Stokes equations for a sphere released from rest. Now, we shall move up just a small step in complexity by changing the solid sphere to a liquid one-to present the so-called Hadamard-Rybczynski solution (e.g. Levich 1962).

\section{Steady Stokes solution for a simple drop}

There are four new and noteworthy aspects of the fluid-fluid problem, vis-a-vis the fluid-solid problem just discussed: (1) The no-slip condition is no longer appropriate, being replaced by a tangential force balance, (2) The terminal velocity is different, (3) It's not at all obvious that the droplet will remain spherical, and (4) Non-constant surface tension induces an additional tangential flow at the interface.

\section{No-slip no longer applies}

The simple BC's for the solid sphere, (28d) - no penetration-and (28e) - no slipare in general replaced by normal and tangential force balances (inside to outside of the drop's surface) beginning with that for constant surface tension:

$$
n\left[P_{i}-P_{0}-\gamma\left(\frac{1}{R_{i}}+\frac{1}{R_{0}}\right)\right]=n \cdot\left[\mu_{i} \tau_{i}-\mu_{0} \tau_{0}\right],
$$

where

$$
\tau_{i} \equiv \nabla \boldsymbol{u}_{i}+\left(\nabla \boldsymbol{u}_{i}\right)^{T} \text { and } \tau_{0}=\nabla \boldsymbol{u}_{0}+\left(\nabla \boldsymbol{u}_{0}\right)^{T}
$$

are the inside and outside strain-rate tensors - and it is worth noting that the famousYoung-Laplace equation is now relegated to a mere portion of a mere $\mathrm{BC}$ for the 
(Navier)-Stokes equations. This is the total (vector) force balance, the normal $\left(\Delta f_{n}\right)$ and tangential portions being

$$
P_{i}-P_{0}-\gamma\left(\frac{1}{R_{i}}+\frac{1}{R_{0}}\right)=n \cdot\left[\mu_{i} \tau_{i}-\mu_{0} \tau_{0}\right] \cdot n
$$

and

$$
0=n \cdot\left[\mu_{i} \tau_{i}-\mu_{0} \tau_{0}\right] \cdot t
$$

respectively, where (in 2D axisymmetric flow), $t$ is a unit tangent vector in the interface.

Specialized to a spherical droplet (assumed for now, verified later-the 'easy' way out), the normal and tangential BCs become: (28d) for the normal, and, from (32), (28d,e), and $(39 b)$,

$$
\mu_{i} \partial\left(u_{\theta}^{i} / r\right) / \partial r=\mu_{0} \partial\left(u_{\theta}^{0} / r\right) / \partial r
$$

at $r=R$ for the tangential $\mathrm{BC}$. [At this point we simply assume that the solution using (28d) will also satisfy (39a); see below.]

These BC's are applied to two sets of Stokes equations (28a thru $28 \mathrm{c}$ ), one for the inside of the drop and one for the outside (host fluid). Using the same method as for the solid sphere (for aiternate methods, see e.g., Leal 1992, Batchelor 1967, or Landau and Lifshitz 1959) gives a 'generalized' version of (31), with $\lambda \equiv \mu_{i} / \mu_{0}$ :

$$
\begin{gathered}
u_{r}^{0}=u_{\infty}\left[1-\frac{3}{2} \cdot \frac{R}{r} \cdot \frac{\lambda+2 / 3}{\lambda+1}+\frac{1}{2}\left(\frac{R}{r}\right)^{3} \cdot \frac{\lambda}{\lambda+1}\right] \cos \theta \\
u_{\theta}^{0}=-u_{\infty}\left[1-\frac{3}{4}\left(\frac{R}{r}\right) \cdot \frac{\lambda+2 / 3}{\lambda+1}-\frac{1}{4}\left(\frac{R}{r}\right)^{3} \cdot \frac{\lambda}{\lambda+1}\right] \sin \theta,
\end{gathered}
$$

with

$$
P_{0}=C_{0}-\rho_{0} g r \cos \theta-\frac{3 \mu_{0} u_{\infty}}{2 R}\left(\frac{R}{r}\right)^{2} \cdot \frac{\lambda+2 / 3}{\lambda+1} \cos \theta
$$

which recovers (31) for $\mu_{i} \rightarrow \infty$.

Also, for the internal flow,

$$
u_{r}^{i}=-u_{\infty}\left[1-(r / R)^{2}\right] \cos \theta \cdot \frac{1}{2(\lambda+1)},
$$




$$
u_{\theta}^{i}=u_{\infty}\left[1-2(r / R)^{2}\right] \sin \theta \cdot \frac{1}{2(\lambda+1)}
$$

and

$$
P_{i}=C_{0}-\rho_{i} g r \cos \theta+\frac{5 \mu_{0} u_{\infty}}{R} \cdot \frac{r}{R} \cos \theta \cdot \frac{\lambda}{\lambda+1}+2 \gamma / R
$$

Fig. 2, from Kim and Karrila (1991), shows a typical set of streamlines $\left(\mu_{i}=\mu_{0}\right)$.

\section{Drag force and terminal speed}

From the outer solution, we obtain in an analogous way as for the solid sphere, except that in this case the normal viscous stress also contributes to the drag (It was zero for the solid sphere.),

$$
F_{D}=\frac{4}{3} \pi R^{3} \rho_{o} g+6 \pi \mu_{o} R u_{\infty} \cdot \frac{2 / 3+\mu_{i} / \mu_{o}}{1+\mu_{i} / \mu_{o}}
$$

for the drag which, when equated to the sphere's weight gives the terminal velocity $\left(u_{\infty} \rightarrow u_{T}\right)$ :

$$
\begin{aligned}
u_{T} & =\frac{2}{9} \frac{g R^{2}}{\mu_{o}}\left(\rho_{i}-\rho_{o}\right) \cdot \frac{1+\mu_{i} / u_{o}}{2 / 3+\mu_{i} / \mu_{o}} \\
& =u_{T}^{s} \cdot \frac{1+\mu_{i} / u_{o}}{2 / 3+\mu_{i} / u_{o}}
\end{aligned}
$$

a weak function of viscosity which recovers $F_{D}$ and $u_{T}^{s}$ for the solid sphere, (35) and (36), as $\mu_{i} \rightarrow \infty$. More noteworthy (because it is new) is the other limit, $\mu_{i} \rightarrow 0$ (the inner fluid becomes more like a gas with $\tau_{r \theta}=0$ ), giving, from the pressure term and the normal viscous stress, $F_{D}=\frac{4}{3} \pi R^{3} \rho_{o} g+4 \pi \mu_{o} R u_{\infty}$ and $u_{T}=\frac{g}{3} \frac{R^{2}}{\mu_{o}}\left(\rho_{i}-\rho_{o}\right)$ which, since $\rho_{o}>\rho_{i}$, describes the steady rise rate of a small gas bubble - $50 \%$ higher than that of a solid with the same density.

It is interesting to re-examine the pressure fields when $u_{\infty}=u_{T}$; from (41c) and (41f) we obtain, using (43),

$$
P_{o}=C_{o}-\rho_{o} g z-\frac{R}{3}\left(\rho_{i}-\rho_{o}\right) g\left(\frac{R}{r}\right)^{2} \cos \theta
$$

and

$$
P_{i}=C_{o}+2 \gamma / R-\rho_{i} g z+\frac{10}{9} R\left(\rho_{i}-\rho_{o}\right) g\left(\frac{r}{R}\right) \cos \theta \cdot \frac{\lambda}{\lambda+\frac{2}{3}},
$$


giving $\left.\quad\left(P_{i}-P_{o}\right)\right|_{R}=\frac{2 \gamma}{R}+\frac{4}{9} \cdot \frac{\lambda-1}{\lambda+2 / 3}\left(\rho_{i}-\rho_{o}\right) g R \cos \theta$,

which merits the following remarks:

1. For $\mu_{i}>\mu_{o}$, the $\Delta P$ at the bottom $(\theta=\pi)$ is less than $2 \gamma / R$ and that at the top is larger than $2 \gamma / R$-because viscous effects caused by flow are stronger than the net buoyancy force. If $\mu_{i}<\mu_{o}$, the opposite is true (buoyancy is stronger).

2. The external pressure is independent of viscosity (as indeed it is also for the falling solid sphere).

3. Whereas $\Delta f_{n}=2 \gamma / R$ is the same as $P_{i}-P_{o}=2 \gamma / R$ for the static case, it is no longer true that the pressure difference is simply $2 \gamma / R$ when there is flow.

4. The exceptional special case is that for $\mu_{i}=\mu_{o}$, for which $\Delta P=2 \gamma / R$ for all $\theta$.

\section{3 . Is the drop really spherical?}

To verify the assumption of a spherical shape requires that the putative solution satisfy exactly the normal stress balance at the interface-(39a) for $R_{i}=R_{o}=R$.

Assuming (still) a spherical shape, (39a) becomes, for the jump in normal stress at $r=R$,

$$
\begin{gathered}
\Delta f_{n} \equiv f_{\text {inside }}^{n}-f_{\text {outside }}^{n} ; \text { i.e. } \\
P_{i}-P_{o}+2\left(\mu_{o} \frac{\partial u_{r}^{o}}{\partial r}-\mu_{i} \frac{\partial u_{r}^{i}}{\partial r}\right)=2 \gamma / R
\end{gathered}
$$

which gives, upon inserting the solution from (41),

$$
C_{i}-C_{o}+\left[\left(\rho_{o}-\rho_{i}\right) g R+\frac{3 \mu_{o} u_{\infty}}{2 R} \cdot \frac{3 \lambda+2}{\lambda+1}\right] \cos \theta=2 \gamma / R
$$

which is only satisfied if both

$$
u_{\infty}=\frac{2 g R^{2}}{9 \mu_{o}}\left(\rho_{i}-\rho_{o}\right) \cdot \frac{(\lambda+1)}{\lambda+2 / 3}
$$


which makes the normal force difference independent of $\theta$, and

$$
C_{i}-C_{o}=2 \gamma / R
$$

which relates the 2 'arbitrary' constants in the pressure fields. Noting that $u_{\infty}=u_{T}$ [cf. (43)] leads to the following important conclusion: The simple droplet in 'free-fall' retains its spherical shape when at its terminal velocity (even for $\gamma \rightarrow 0$ !). "For virtually any other problem involving the motion of bubbles or drops in a viscous fluid, the shape would be nonspherical"-Leal 1992, p. 212). It will turn out, however, that God is good and, as we shall point out later, the drop actually remains spherical during the transition period that is described by the transient Stokes equations (We will, of course still need $\mathrm{Re} \cong 0-\boldsymbol{u} \cdot \nabla \boldsymbol{u}$ negligible).

\section{Non-constant surface tension: Marangoni flow}

If $\gamma$ is not independent of temperature and/or concentration, the tangential stress balance, given thus far by (39b), needs another term because a variable $\gamma$ along the surfaces produces a variable shear stress there. Thus, (39b) and (40) must be generalized to (for a sphere-for simplicity; thus not fully general)

$$
\mu_{i}\left[\frac{\partial u_{\theta}^{i}}{\partial r}-u_{\theta}^{i} / r\right]-\mu_{\circ}\left[\frac{\partial u_{\theta}^{o}}{\partial r}-u_{\theta}^{o} / r\right]=\frac{1}{r} \frac{\partial \gamma}{\partial \theta}
$$

at $r=R$, and we have (still) assumed axisymmetry; the jump in shear stress is proportional to the surface tension gradient-which itself is (usually) nonzero only by virtue of a surface concentration or temperature gradient. As the former is probably more important for our situation, we write $\gamma=\gamma(C)$ and $\partial \gamma / \partial \theta=\partial \gamma / \partial C \cdot \partial C / \partial \theta$, where $C$ represents concentration of some solute, and it is often true that $\gamma(C)=\gamma_{o}\left[1-\alpha\left(C-C_{o}\right)\right]$; i.e., a linear variation is a good approximation.

But we take it no further-for now. All that can be said in general is that a variable $\gamma$ will cause the velocity fields in both drop and host to differ from those very special solutions that permitted a spherical shape. Thus, the Marangoni effect (flows caused by non-constant $\gamma$ ) can lead to a loss of sphericity and is thus to be avoided if possible. Actually, for $\alpha\left(C-C_{0}\right) \ll 1$, Edwards et al. (1991) show that the droplet will still remain spherical-at a slightly smaller terminal velocity. 


\section{Steady Stokes Flow for a Compound/Encapsulated Drop}

This last case, while much more appropriate than those previously considered, is also very much more difficult-depending as it does on 2 densities and 3 viscosities, the radius ratio $\left(R_{c} / R_{s}\right)$, and the eccentricity/offset. Thus, it is not suprising that closed form solutions are not to be found. Hence, we shall content ourselves in providing just a brief summary of those few relevant results that we have found in the literature. Fig. 3 shows a qualitative sketch of the flow field for such a drop.

On the assumption that the core is concentric and thus motionless (no translation) relative to the shell, Brunn and Roden (1985) obtained the potentially important result that both core and shell are still perfect spheres (for droplets at their terminal velocity and $\mathrm{Re}=0$ ) - -a result that, like the simple drop, even holds true for $\gamma_{c}$ and $\gamma_{s} \rightarrow 0$.

Another of their conclusions-which is suspicicious owing to their (untested) assumption that concentricity prevails-is this: In order that a solution of the equations of motion of core and shell exist (at terminal velocity), it is only necessary that $\left(\rho_{s}-\rho_{H}\right) /\left(\rho_{c}-\rho_{s}\right)<0$; i.e., $\rho_{s}-\rho_{H}<0 \Leftrightarrow \rho_{c}-\rho_{s}>0$, a result they themselves call "astonishing."

In an earlier paper employing the same assumptions (concentric spheres with no relative translational velocity between core and shell), Rushton and Davies (1983) obtained the following result for terminal velocity: $u_{\text {solid }}<u_{\text {compound }}<u_{\text {simple }}$ which, when combined with other known results, leads to the following 'cascade' of inequalities for terminal speeds (at equal $\Delta \rho$ ):

$$
\frac{2}{3} u_{\text {bubble }}=u_{\text {solid }}<u_{\text {compound }}<u_{\text {simple }}<u_{\text {bubble }}=\frac{3}{2} u_{\text {solid }},
$$

where "bubble" means $\mu_{i}=0$. The range of terminal speeds is, somewhat surprisingly, rather narrow. This 'reasons' for these inequalities are as follows:

(i) $u_{\text {compound }}>u_{\text {solid }}$ because the largest drag is that from the no-slip (solid) boundary condition. The non-zero tangential velocity at the liquid-liquid (shell-host) interface reduces the shear stress there.

(ii) $u_{\text {compound }}<u_{\text {simple }}$ because flow in the core necessarily reduces shell flow which reduces the tangential velocity at the shell-host interface. 
(iii) $u_{\text {simple }}<u_{\text {bubilc }}$ because a stress-free (bubble) tangential condition results in the largest tangential velocity and smallest shear stress.

Another important result 'discovered' by these authors and since verified by others is this: For $R_{c} \rightarrow R_{s}$, the 'reversed' flow in the shell (see Fig. 3) causes the viscous shear stresses to become so large that all flow ceases in both shell and core; a thin-walled compound droplet (with concentric core) behaves much like a solid sphere-a result of which is that lubrication theory (Stokes flow, basically; see, e.g. Batchelor 1967) will apply in both core and shell even for finite Reynolds number-as long as the shell is sufficiently thin.

In Sadhal and Oguz (1985), which is rather more general in that, while they permit/consider both eccentric locations of core-in-shell and determine under what conditions the core remains (translationally) motionless with respect to the shell (equilibrium configurations) at terminal velocity, they do so only with the assumption of perfect sphericity for core and shell (e.g. large surface tensions, small drops)-thus leading to more believable results than those of their predecessors. Under these conditions, their salient results of most interest to us are as follows (see too Fig. 4):

1. If $\rho_{c} V_{c}+\rho_{s} V_{s}<\rho_{H}\left(V_{c}+V_{s}\right)$ where $V=$ volume, the rising droplet can be in equilibrium (both core and shell translating upward at the same terminal velocity) if and only if $\rho_{c}>\rho_{s}$ because the shear stress (and pressure, probably) on the core is trying to lift the core relative to the shell. The stable orientation is then one in which the core sits toward the front of the rising shell; i.e. it is not concentric.

2. If $\rho_{c} V_{c}+\rho_{s} V_{s}>\rho_{H}\left(V_{c}+V_{s}\right)$, the falling droplet can only be in equilibrium if the core is less dense than the shell because the shear stress (and pressure, probably) from the shell is trying to pull the core downward. Under these conditions, the stable orientation has the core situated toward the rear of the falling shell. (I.e., in both stable motions, the core's center is located above the center of the shell. This latter case was also observed in the laboratory (Hamilton et al., 1997).

3. If $\rho_{c}=\rho_{s}\left(\neq \rho_{H}\right)$ in either of the above two cases, the motion is actually unstable-relative translation of the core must occur.

4. The stable solutions in 1) and 2) above are actually only realizable for specific ranges of both physical properties and radius ratio, $R_{c} / R_{s}$. A stable steady Stokes solution of $\mathrm{a}^{-}$ compound droplet with unmatched densities is, in general, not easy to obtain! This is 
(presumably) one reason that most target fabrication efforts via encapsulation to date have used time-dependent fluid dynamics-a subject we shall soon turn to.

\section{E. Droplet shape for $R e>0$,}

This section too, is mostly a brief literature review. We will summarize the available knowledge that examines the nonlinear effects that accompany the case of $\operatorname{Re}>0$-via perturbation analyses, first for simple drops and then for compound drops.

\section{Simple drops in free-fall}

Taylor and Acrivos (1964), in what has been called a "classic" paper, broke the nonlinear ground via singular perturbation analysis-not long after the method had first been discovered/invented by Proudman and Pearson (1957). The analysis is (necessarily) long and complicated and we shall therefore skip the details. By examining the normal stress balance at the interface at terminal speed, which is now not independent of $\theta$, they obtained the perturbed shape of the drop by finding $\zeta(\theta)$ in [cf. (7)]

$$
r(\theta)=R[1-\zeta(\theta)]
$$

where $R$ is the equivalent (same volume) spherical radius, as

$$
\zeta=f\left(\mu_{i} / \mu_{o} \equiv \mu, \rho_{i} / \rho_{o} \equiv \rho\right) \cdot \rho u_{T}^{2} R / \gamma \cdot P_{2}(\cos \theta)
$$

where $f(\mu, \rho) \equiv \frac{1}{4(\mu+1)^{2}}\left[\frac{81}{80} \mu^{3}+\frac{57}{20} \mu^{2}+\frac{103}{40} \mu+\frac{3}{4}-(\mu+1) \frac{\rho-1}{12}\right]$,

which is a very wcak function of $\mu$ and $\rho$, varying only from $\sim 0.21$ for a gaseous interior $(\mu, \rho \rightarrow 0$, a bubble) to $\sim 0.25$ for a solid interior $(\mu \rightarrow \infty)$. These authors also give the next term in the series expansion [to $O\left(u^{3} P_{3}(\theta)\right)$ ] and Brignell (1973) gives the next two after these-none of which we feel are needed in our mostly 'first-order' analysis. Noting that $P_{2}(\pi / 2)=-1 / 2$ and $P_{2}(0)=1$ shows that the droplet shape is basically that of an oblate spheroid - and the result is only valid for $\operatorname{Re}<O(1)$ and $\rho_{\mathrm{o}} u_{T}^{2} R / \gamma<<1$; i.e.;

$C a \cdot \operatorname{Re}<O(1)$, where $\operatorname{Re} \equiv u_{T} R / v_{o}$ and $C a \equiv \mu_{o} u_{T} / \gamma$ is the cavitation number (and $C a \cdot \operatorname{Re}=W e$, the Weber number; $\left.W e=\rho_{o} u_{T}^{2} R / \gamma\right)$. Review: $\operatorname{Re}<1 \Rightarrow$ inertial forces < viscous forces and $\mathrm{Ca}<1 \Rightarrow$ viscous forces $<$ surface tension forces (both of which together $\Rightarrow \zeta \ll<1$. 
To the same order of approximation, the terminal velocity is

$$
u_{T}=u_{T}^{s} \cdot \frac{1+\mu_{i} / \mu_{o}}{2 / 3+\mu_{i} / \mu_{o}} \cdot\left[1-\frac{3}{8} \frac{u_{T}^{s} R}{v}\right]+O\left(u_{T}^{s}\right)^{3},
$$

where $u_{T}^{s}$ is the solid sphere's terminal velocity-cf. (36) and (43); and we must have $u_{s}^{T} R / v<<1$; the nonlinear terms cause a reduction in the terminal speed (a larger drag coefficient) from that for $\mathrm{Re}=0$. Note that (57) corresponds approximately to the Oseen correction to the Stokes flow result for a simple $\left(\mu_{i}=\infty\right)$ drop and we point out that using the geometric mean of the Stokes drag and the Oseen drag, at least for solid spheres, generates a pretty accurate result for Re up to 50 or so and is probably better than (57) even for a simple drop:

$$
u_{T}=u_{T}^{s} \cdot \frac{1+\mu_{i} / \mu_{o}}{2 / 3+\mu_{i} / \mu_{o}} / \sqrt{1+\frac{3}{8} \frac{u_{T}^{s} R}{v}},
$$

which is about $2 \%$ low at $\frac{u_{T}^{s} R}{v}=50$ and $\mu_{i}=\infty$. (The Oseen approximation, (57), for the same case is a factor of about 4.5 too low and the Stokes solution a factor of $\sim 4.4$ too high.)

Inserting $u_{T}$ from (57) into (55), and $\zeta(\theta)$ from (55) into (54) gives the departure from sphericity [cf. (20)]:

$$
M o o R=3 f(\mu, \rho) \cdot \rho_{o} u_{T}^{2} R^{2} / \gamma
$$

where $f \cong 0.23$. It is worthwhile noting that this result, combined with (57) and (36), shows that, to zeroth order (Stokes; omit the $3 / 8$ term)

$$
M o o R \cong \frac{4}{27} f(\mu, \rho)\left(\frac{1+\mu_{i} / \mu_{o}}{2 / 3+\mu_{i} / \mu_{o}}\right)^{2} \frac{\rho_{o}}{\gamma}\left(\frac{g \Delta \rho}{\mu_{o}}\right)^{2} R^{6} ;
$$

i.e., it varies with the 6 th power of the terminal speed! [For $\operatorname{Re}>0$ the R-dependence is somewhat less-perhaps $\left.O\left(R^{4-5}\right)\right]$. This result alone helps to explain why large drops are much more difficult to keep spherical than small ones-at least in a free-fall mode.

\section{Simple drop in a linear shear flow}

Another variation on the droplet deformation in a moving medium was provided by Barthès-Biesel and Acrivos (1973); this time for a droplet "suspended" in a general linear 
shear field. Although they were primarily interested in large deformations and droplet breakup, they do supply some results that are of interest here. Specifically, for a given shear rate, $G(\cong \Delta u / \Delta x)$, their Fig. 6 shows that

$$
M o o R \equiv 4 \varepsilon R \equiv 4 \mu_{o} G R^{2} / \gamma
$$

where, for our purposes, $G$ may be related to a rotation rate, $\omega=2 \pi f$, and a container radius, $r_{c}$, via $G \cong\left(\omega r_{c}\right) / r_{c}=\omega=2 \pi f$.

\section{Compound drop in free-fall}

Returning to Brunn and Roden (1985), these authors also obtained a linearized result (small $\mathrm{Re}$ ) for shell deformation-again under the same, rather stringent assumption that the core remains at the center of the shell. They then, in a sense, generalized the TaylorAcrivos results for a simple droplet to a (concentric) compound droplet. With respect to droplet shape, their key results are the following:

(1) for $\operatorname{Re}=0$, the compound droplet remains spherical, even if the surface tensions tend toward zero.

(2) The shell outer surface deforms into an oblate spheroid ( $P_{2}$-mode, as usual), like a simple drop.

(3) The core deforms into a ( $P_{2}$-mode) prolate spheroid.

(4) Thus, the shell thickness is a minimum at the poles and a maximum at the equator.

(5) The magnitude of each perturbation is again proportional to the appropriate Weber number, $\rho_{j} u_{T}^{2} R_{j} / \gamma_{j}$, which again must be small for the results to be valid.

\section{Unsteady Hydrodynamics}

For all time-dependent motion, the starting point is (25) and the time-dependent version of (24):

$$
\frac{\partial u}{\partial t}+\boldsymbol{u} \cdot \nabla \boldsymbol{u}+\nabla(P / \rho)=v \nabla^{2} \boldsymbol{u}+f
$$

which, when $\boldsymbol{u} \cdot \nabla \boldsymbol{u}$ is small enough to be neglected (Re $\ll 1$ ), gives the transient Stokes equations. A completely 'opposite' assumption-that (only) the viscous term, $v \nabla^{2} u$, is 
negligible-gives the Euler equations. And this, plus one more sometimes-valid assumption-irrotational/potential flow-leads to the 'ideal' flow momentum conservation equation (with the body force neglected)

$$
\frac{\partial u}{\partial t}+\nabla\left(P / \rho+\frac{1}{2} q^{2}\right)=\mathbf{0}
$$

where $q=|\boldsymbol{u}|$ and we have used the vector identity $\boldsymbol{u} \cdot \nabla \boldsymbol{u}=\frac{1}{2} \nabla(\boldsymbol{u} \cdot \boldsymbol{u})-\boldsymbol{u} \times(\nabla \times \boldsymbol{u})$ with $\nabla \times u=0$ (irrotational). The quantity $P_{T} \equiv P+\frac{1}{2} \rho q^{2}$ is the total, or Bernoulli, pressure.

Since $\boldsymbol{u}$ is curl-free when (63) applies, we can define a scalar $\phi$, the velocity potential, via

$$
\boldsymbol{u} \equiv \nabla \phi
$$

Equation (25) then gives

$$
\nabla^{2} \phi=0
$$

and (63) gives

$$
\frac{\partial \phi}{\partial t}+P / \rho+\frac{1}{2} q^{2}=F(t)
$$

which, when the arbitrary function of time, $F(t)$, is set to zero, is the equation for the pressure. Thus, given appropriate boundary conditions, the solution of Laplaces' equation, (65), gives the potential from which (64) gives the velocity and (66) the pressure:

$$
P=-\rho\left(\frac{\partial \phi}{\partial t}+\frac{1}{2} q^{2}\right) .
$$

The transient Stokes equations will be of use in estimating the time required to attain terminal velocity - and the ideal/potential flow equations will be useful for estimating the frequency of capillary/surface waves and the pressure forces on core and shell under transient conditions of accelerated flow. Both extremes will be used to estimate the viscous damping rate of capillary waves and, finally, for approximating the 'bulk'/global equations of (translational) motion for both core and shell. 


\section{A. Capillary (surface tension) waves}

In response to the question, "How does a droplet (simple or compound) respond to a given perturbation from an equilibrium (spherical) shape?," Saffren et al. (1982) studied an ideal compound (and simple) drop and Miller and Scriven (1968) examined a viscous simple drop-whose results we shall summarize below.

\section{Ideal waves, simple and compound drops}

In an early and important fluid dynamics paper on droplet dynamics that probably deserves wider dissemination, Saffren et al. (1981) determined the 'normal modes' (capillary surface waves) that could exist at the two interfaces between the 3 inviscid fluids comprising an ideal (concentric) compound drop. The normal mode analysis is an eigenvalue problem, obtained by assuming time-dependence of the form $e^{i \omega t}$ and linearizing the resulting equations, where $\omega$ (the wave frequency) is the eigenvalue and the concomitant surface shape(s) the eigenvector. The solution [of (63), basically] is obtained in terms of spherical harmonics, and the results are:

\section{(i) Simple drop (not a new result, of course)}

$$
\omega_{n}^{I}=\sqrt{\frac{\gamma}{R^{3}} \cdot \frac{(n-1) n(n+1)(n+2)}{n \rho_{o}+(n+1) \rho_{i}}},
$$

where $\omega_{n}^{l}$ is the (inviscid) frequency of the $n-t h$ mode. This result, at least for $\rho_{o}=0$, is well-known; see, e.g. Landau and Lifshitz (1959), Chandrasekar (1961). For $\rho_{o} \neq 0$, it is derived in Lamb (1945). For $n=2$, the mode of most interest $[n=0$ is a purely radial mode, precluded by incompressibility (Landau and Lifshitz 1959) and $n=1$ corresponds to pure translation] and $\rho_{o} \cong \rho_{i}$,

$$
\omega_{2}^{I} \cong \sqrt{\frac{4.8 \gamma}{\rho R^{3}}} .
$$

(ii) Compound drop (a new result)

$$
\omega_{ \pm}^{(n)}=\left\{\frac{\sqrt{\frac{\gamma_{c} \gamma_{s}}{R_{c}^{3} R_{s}^{3}}} \cdot \frac{(n-1) n(n+1)(n+2)}{(2 n+1) \rho_{s}} \cdot\left[A_{n} \pm \sqrt{B_{n}}\right]}{\left[1+\frac{n\left(\rho_{H}-\rho_{s}\right)}{(2 n+1) \rho_{s}}\right] \cdot\left[1+\frac{(n+1)\left(\rho_{c}-\rho_{s}\right)}{(2 n+1) \rho_{s}}\right]\left(\frac{R_{s}}{R_{c}}\right)^{n+1 / 2}-C_{n}\left(\frac{R_{c}}{R_{s}}\right)^{n+1 / 2}}\right\}^{1 / 2},
$$




$$
\begin{aligned}
& \text { where } A_{n} \equiv \frac{1}{2}\left[\frac{R_{c}}{R_{s}} \sqrt{\frac{\gamma_{s} R_{c}}{\gamma_{c} R_{s}}} \cdot M_{i}^{(n)}+\frac{R_{s}}{R_{c}} \sqrt{\frac{\gamma_{c} R_{s}}{\gamma_{s} R_{c}}} \cdot M_{o}^{(n)}\right], \\
& B_{n} \equiv 1+\frac{1}{4}\left[\frac{R_{c}}{R_{s}} \sqrt{\frac{\gamma_{s} R_{c}}{\gamma_{c} R_{s}}} \cdot M_{i}^{(n)}-\frac{R_{s}}{R_{c}} \sqrt{\frac{\gamma_{c} R_{s}}{\gamma_{s} R_{c}}} \cdot M_{o}^{(n)}\right]^{2}, \\
& \text { where } \quad M_{i}^{(n)} \equiv {\left[1+\frac{(n+1)\left(\rho_{c}-\rho_{s}\right)}{(2 n+1) \rho_{s}}\right]\left(\frac{R_{s}}{R_{c}}\right)^{n+1 / 2}-\frac{(n+1)\left(\rho_{c}-\rho_{s}\right)}{(2 n+1) \rho_{s}}\left(\frac{R_{c}}{R_{s}}\right)^{n+1 / 2}, } \\
& M_{o}^{(n)} \equiv {\left[1+\frac{n\left(\rho_{H}-\rho_{s}\right)}{(2 n+1) \rho_{s}}\right]\left(\frac{R_{s}}{R_{c}}\right)^{n+1 / 2}-\frac{n\left(\rho_{H}-\rho_{s}\right)}{(2 n+1) \rho_{s}}\left(\frac{R_{c}}{R_{s}}\right)^{n+1 / 2}, } \\
& \text { and } C_{n} \equiv \frac{(n+1)\left(\rho_{c}-\rho_{s}\right)}{(2 n+1) \rho_{s}} \cdot \frac{n\left(\rho_{H}-\rho_{s}\right)}{(2 n+1) \rho_{s}},
\end{aligned}
$$

and we admit to not checking their 'algebra'. For the thin-shell limit $\left(R_{c} \approx R_{s}\right)$ the frequency is simply that given by (68) with $\gamma$ replaced by $\gamma_{c}+\gamma_{s}$; two surfaces, two surface tensions, higher frequency (bubble mode only, of course).

The high-frequency mode (for each $n$ ) is called the "bubble" mode, in which core and shell oscillate in-phase. The low-frequency out-of-phase mode, $\omega_{-}$, is called the "sloshing" mode, which involves significantly more 'tangential' flow than does the bubble mode. For the principle (low) mode of interest, $n=2\left(P_{2}\right.$ mode), these 'simplify' to

$$
\omega_{ \pm}^{(2)}=\left\{\frac{\frac{4.8}{\rho_{s}} \sqrt{\frac{\gamma_{c} \gamma_{s}}{R_{c}^{3} R_{s}^{3}}}\left(A_{2} \pm \sqrt{B_{2}}\right)}{\left[1+\frac{0.4\left(\rho_{H}-\rho_{s}\right)}{\rho_{s}}\right]\left(1+\frac{0.6\left(\rho_{c}-\rho_{s}\right)}{\rho_{s}}\right)\left(\frac{R_{s}}{R_{c}}\right)^{3 / 2}-C_{2}\left(\frac{R_{c}}{R_{s}}\right)^{5 / 2}}\right\}^{1 / 2},
$$

where now

and

$$
M_{i}^{(2)}=\left[1+\frac{0.6\left(\rho_{c}-\rho_{s}\right)}{\rho_{s}}\right]\left(\frac{R_{s}}{R_{c}}\right)^{5 / 2}-0.6 \frac{\left(\rho_{c}-\rho_{s}\right)}{\rho_{s}}\left(\frac{R_{c}}{R_{s}}\right)^{5 / 2}
$$

$$
M_{o}^{(2)}=\left[1+\frac{0.4\left(\rho_{H}-\rho_{s}\right)}{\rho_{s}}\right]\left(\frac{R_{s}}{R_{c}}\right)^{5 / 2}-0.4 \frac{\left(\rho_{H}-\rho_{s}\right)}{\rho_{s}}\left(\frac{R_{c}}{R_{s}}\right)^{5 / 2} \text {. }
$$

Fig. 5, taken from the above reference, shows the two modes for an oil (silicone)/water/oil droplet. 


\section{Simple viscous drop}

For the (non-simple) case of a simple viscous drop, Miller and Scriven (1968) solved the eigenproblem obtained from the transient Stokes equations, via $e^{i \omega x} \cdot e^{-t / \tau}$ time-dependence, where $\tau$ is the viscous damping time constant, with the result of most interest here being that for finite but 'small' viscosities (for 'large' viscosities, they predict a monotonic decay; i.e. no oscillations):

$$
\begin{aligned}
\omega_{n} & =\omega_{n}^{l}-\frac{(2 n+1)^{2} \sqrt{\omega_{n}^{l} \mu_{i} \mu_{o} \rho_{i} \rho_{o}}}{2 R \sqrt{2}\left[n \rho_{o}+(n+1) \rho_{i}\right]\left[\sqrt{\mu_{i} \rho_{i}}+\sqrt{\mu_{o} \rho_{o}}\right]}, \\
& \equiv \omega_{n}^{I}-\Delta \omega_{n},
\end{aligned}
$$

where $\omega_{n}^{I}$ is the frictionless frequency, given by (68), and the decay rate, $\lambda \equiv \tau^{-1}$, is given by

$$
\tau^{-1}=\Delta \omega_{n}+\frac{(2 n+1)\left\{2\left(n^{2}-1\right) \mu_{i}^{2} \rho_{i}+2 n(n+2) \mu_{o}^{2} \rho_{o}+\mu_{i} \mu_{o}\left[\rho_{i}(n+2)-\rho_{o}(n-1)\right]\right\}}{2 R^{2}\left[n \rho_{o}+(n+1) \rho_{i}\right]\left[\sqrt{\mu_{i} \rho_{i}}+\sqrt{\mu_{o} \rho_{o}}\right]^{2}},
$$

the first term coming from a boundary layer effect at $r=R$ and the second corresponding to dissipation outside of the boundary layer [whose thickness $\left.\cong\left(\sqrt{v / \omega_{n}}\right)\right]$.

For the $n=2$ mode and $\rho_{i} \cong \rho_{o}$,

$$
\Delta \omega_{2}=\frac{2.5 \sqrt{\omega_{2}^{I} \mu_{i} \mu_{o}}}{R \sqrt{2 \rho}\left(\sqrt{\mu_{i}}+\sqrt{\mu_{o}}\right)}
$$

and

$$
\tau^{-1}=\Delta \omega_{2}+\frac{6 \mu_{i}^{2}+16 \mu_{o}^{2}+3 \mu_{i} \mu_{o}}{2 \rho R^{2}\left(\sqrt{\mu_{i}}+\sqrt{\mu_{o}}\right)^{2}}
$$

which are the results we shall use.

Remark: In a paper discovered during the final typing of this paper, Basaran et al. (1989) show an additional term, overlooked by Miller and Scriven, that reduces $\tau^{-1}$ in (73). For our parameters, however, the change is negigible-although further work in the area should probably incorporate these newer results in more detail. 


\section{Compound viscous drop}

Landman (1985) performed a 'partial' analysis of a compound drop, accounting for viscous effects in core and shell but not in the host. (The compound drop is in a gas, or a vacuum). Also not accounted for was fluid acceleration; i.e. she set $\partial u / \partial t=\mathbf{0}$ to obtain the steady Stokes equations, with the only time-dependence then being that of the free surface shape. With these assumptions, she found that perturbations are always overdamped-no oscillations. Also, the dominant mode $(n=2)$ displayed out-of-phase behavior between core and shell-the sloshing mode. The bubble mode decays more quickly. These results are, in a sense, opposite those from Saffren et al. (1981) for the inviscid case and, since they seem to predict no oscillations for virtually any values of $\mu_{i}$ and $\mu_{o}$, they are somewhat suspect. Clearly the neglect of the acceleration term in the Stokes equations can only be sometimes justified (and was not done by Miller and Scriven who, for the simple drop, obtained the more reasonable results of sometimes underdamped and sometimes overdamped oscillatory decay-depending on the properties). E.g., if $\tau \equiv R^{2} / V$ is the viscous decay time constant, the steady-state assumption would seem to require $\tau<<T=2 \pi \sqrt{\rho R^{3} / 4.8 \gamma}$-the period of oscillation, from (69). This $\Rightarrow R \ll 4 \pi^{2} \rho v^{2} / 4.8 \gamma$ which, for our nominal parameter values ( $\rho=1, v=.01, \gamma=10$-all in cgs units), gives $R \ll 0.8 \mu m-$ a very tiny drop! Using $v=1$ as a guess for the shell's viscosity, increases this to $R \ll 0.8 \mathrm{~cm}$ which just might cover our case. On the other hand, if the viscous time constant is based on the shell thickness rather than on the radius-which may actually be more reasonable, $\tau<<T$ leads to $\Delta R=R_{s}-R_{c}<<\left[2 \pi v \sqrt{\rho R^{3} / 4.8 \gamma}\right]^{1 / 2}$ which, for the above parameters and $R=0.1 \mathrm{~cm}$, yields $\Delta R<<170 \mu \mathrm{m}$, which is closer being satisfied for the case of interest. Replacing $v=.01$ by $v=1$ increases it even further- to $\Delta R<<1.7 \mathrm{~cm}$, which is surely satisfied.

Perhaps we should be content just to draw the conclusion, from Miller and Scriven and from Landman, that any oscillations of a compound drop will be very quickly damped by viscosity-the calculations of which for the viscous drop will be presented in more detail later, under "Applications".

\section{B. Time-dependent potential flow, concentric spheres}

As mentioned already, the theory of time-dependent potential flow (inviscid, irrotational) is useful for estimating some of the additional forces felt by core and shell during time-varying situations. In this section we will generate a simple potential flow 'model' which, while definitely quite restrictive in application owing to some stringent 
assumptions needed to derive it, may actually be quite useful, partly because of the additional insight that it provides. Thus, for the special case of concentric configurations, we shall first examine the potential flow and (especially) accelerating potential flow between core and shell, followed by that in the core and the host-and point out one advantage accruing from thin shells.

\section{Potential flow in the shell}

Suppose, instantaneously, that the z-component of the core velocity $\left(u_{c}\right)$ is given, as is that of the shell $\left(u_{s}\right)$-in the concentric configuration. The potential flow in the shell for this case is governed by (with $\boldsymbol{u} \equiv \nabla \phi$ )

$$
\nabla^{2} \phi=\frac{1}{r^{2}}\left[\frac{\partial}{\partial r}\left(r^{2} \frac{\partial \phi}{\partial r}\right)+\frac{1}{\sin \theta} \frac{\partial}{\partial \theta}\left(\sin \theta \frac{\partial \phi}{\partial \theta}\right)\right]=0 \quad \text { in } R_{c}<r<R_{s},
$$

with $\partial \phi / \partial r=u_{c} \cos \theta$ at $r=R_{c}$ and $\partial \phi / \partial r=u_{s} \cos \theta$ at $r=R_{s}$.

The solution for the potential field is

$$
\phi=\frac{\left(u_{s} R_{s}^{3}-u_{c} R_{c}^{3}\right) r+\frac{\left(u_{s}-u_{c}\right) R_{s}^{3} R_{c}^{3}}{2 r^{2}}}{R_{s}^{3}-R_{c}^{3}} \cdot \cos \theta
$$

from which the velocities may be obtained:

$$
u_{r}=\frac{\partial \phi}{\partial r}=\frac{\left(u_{s} R_{s}^{3}-u_{c} R_{c}^{3}\right)-\frac{\left(u_{s}-u_{c}\right) R_{s}^{3} R_{c}^{3}}{r^{3}}}{R_{s}^{3}-R_{c}^{3}} \cdot \cos \theta
$$

and $u_{\theta}=\frac{1}{r} \frac{\partial \phi}{\partial \theta}=-\frac{\left(u_{s} R_{s}^{3}-u_{c} R_{c}^{3}\right)+\frac{\left(u_{s}-u_{c}\right) R_{s}^{3} R_{c}^{3}}{2 r^{3}}}{R_{s}^{3}-R_{c}^{3}} \cdot \sin \theta$

More important for our purposes, however, is the pressure induced by a timedependent flow-since the potential flow velocity, (79) and (80), contributes nothing to the drag force (per d'Alembert and his famous paradox). Thus, considering $u_{s}$ and $u_{c}$ to be time-dependent (and, for the time being, given), we use (67) to obtain the acceleration portion $\left(P_{s}^{A}\right)$ of the shell pressure field, viz., 


$$
P_{s}^{A} \equiv-\rho_{s} \partial \phi / \partial t=-\rho_{s}\left[\frac{\left(\dot{u}_{s} R_{s}^{3}-\dot{u}_{c} R_{c}^{3}\right) r+\frac{\left(\dot{u}_{s}-\dot{u}_{c}\right) R_{s}^{3} R_{c}^{3}}{2 r^{2}}}{R_{s}^{3}-R_{c}^{3}}\right] \cos \theta
$$

which, when evaluated at $r=R_{c}$ gives the pressure force on the core (by the shell) and when evaluated at $r=R_{s}$ gives the pressure force on the host (by the shell), only the first of which we will actually use-as follows:

Recalling that pressure acts only normal to any boundary, the force on the core in the flow direction $(z)$ caused by $P_{s}^{A}$ is $-P_{s}^{A}\left(R_{c}, \theta\right) \cos \theta$, and $-P_{s}^{A}\left(R_{c}, \theta\right) \cos \theta\left(R_{c} \sin \theta d \theta\right)\left(R_{c} d \phi\right)$ integrated over the surface of the (inner) sphere gives the 'drag' force:

$$
\begin{aligned}
& F_{D}^{A}=R_{c}^{2} \int_{o}^{2 \pi} \int_{o}^{\pi}-P_{s}^{A}\left(R_{c}, \theta\right) \cos \theta \sin \theta d \theta d \phi \\
& =2 \pi R_{c}^{2} \rho_{s}\left[\frac{\left(\dot{u}_{s} R_{s}^{3}-\dot{u}_{c} R_{c}^{3}\right) R_{c}+\frac{\left(\dot{u}_{s}-\dot{u}_{c}\right) R_{s}^{3} R_{c}}{2}}{R_{s}^{3}-R_{c}^{3}}\right] \int_{o}^{\pi} \cos ^{2} \theta \sin \theta d \theta \\
& =\frac{4}{3} \pi R_{c}^{3} \rho_{s} \cdot \frac{\frac{3}{2} R_{s}^{3} \dot{u}_{s}-\left(R_{c}^{3}+\frac{1}{2} R_{s}^{3}\right) \dot{u}_{c}}{R_{s}^{3}-R_{c}^{3}},
\end{aligned}
$$

a result that we shall use later in the equations of motion.

Fig. 6 shows the pressure isobars for the case $\dot{u}_{s}=1, \dot{u}_{c}=0, \rho_{s}=1, R_{s}=1$ and $R_{c}=0.9$; the shell is accelerated toward a motionless core-and we emphasize that this is only a 'snapshot', i.e. it only applies instantaneously, since relative core-shell motion must occur for $t>0$. Fig 6 also shows the corresponding streamlines $(\psi)$-here for $u_{o}=1, u_{i}=0$-which of course neglects any relative motion between spheres. [It corresponds to the instantaneous potential flow given by (78)].

\section{Potential flow in the core and the host}

Similar, and simpler analyses can be applied to both flow in the core and flow in the host-using (of course) the same boundary conditions at $r=R_{c}$ and $r=R_{s}$; viz. (77).

The results are 


$$
\begin{aligned}
\phi_{c} & =u_{c} r \cos \theta, \\
P_{c}^{A} & \equiv-\rho_{c} \frac{\partial \phi_{c}}{\partial t}=-\rho_{c} \dot{u}_{c} r \cos \theta, \\
\phi_{H} & =\left[u_{H} r+\frac{\left(u_{H}-u_{s}\right) R_{s}^{3}}{2 r^{2}}\right] \cos \theta, \\
\text { and } \quad P_{H}^{A} & =-\rho_{H} \frac{\partial \phi_{H}}{\partial t}=-\rho_{H}\left[\dot{u}_{H} r+\frac{\left(\dot{u}_{H}-\dot{u}_{s}\right) R_{s}^{3}}{2 r^{2}}\right] \cos \theta,
\end{aligned}
$$

where we will be interested in $P_{c}^{A}\left(r=R_{c}\right)$, which gives the force on the shell's inner surface caused by an accelerating core-and in $P_{H}^{A}\left(r=R_{s}\right)$ which gives the force on the shell's outer surface caused by an accelerating host fluid, both integrated as above for $F_{D}^{A}$.

Before actual utilization of these results, a few clarifying remarks may be in order:

1. Even though this is a potential flow analysis, the resulting pressure forces are also present in the actual, viscous, case; i.e., accelerations at the boundaries cause potential flow forces in the full Navier-Stokes equations.

2. The first term $(\sim r)$ in each pressure equation corresponds to simple 'frec-stream' or 'solid body' bulk acceleration; i.e. it corresponds to

$$
\partial P / \partial z=-\rho \dot{u}, \text { giving } P=-\rho \dot{u} z=-\rho \dot{u} r \cos \theta,
$$

where the same force in (81) is merely 'complicated by the geometry'-and by the existence of two separate 'free-stream' accelerations.

3. The second terms in the pressure equations (those $\sim 1 / r^{2}$ ), always involving differences in accelerations, will be seen to be manifestations of the so-called "added mass" effect that always occurs when an object is accelerated through a fluid-the details (and fuller appreciation of which) will show-up later, when we write the equations of motion.

4. The pressure in the shell (and the force on the core) becomes quite large in the case of a thin shell [this, paradoxically perhaps, in spite of the (still true) fact that the velocities in both core and shell tend toward zero when $R_{c} \rightarrow R_{s}$-as

discussed earlier.] For $R_{c} \rightarrow R_{s}$, (81) becomes (for $R_{c} \approx R_{s} \equiv R$ and $\delta R \equiv R_{s}-R_{c}$ ) 


$$
P_{s}^{A} \cong \frac{-\rho_{s} \cos \theta}{3}\left[r+\frac{1}{2} \frac{R}{r^{2}}\right]\left(\frac{R}{\delta R}\right)\left(i_{s}-i_{c}\right)
$$

the difference in accelerations is amplified by a factor that $\rightarrow \infty$ as $\delta R \rightarrow 0-$ a result that will soon be seen to be potentially significant (because it shows that the core tends to 'follow' the shell).

5. An interesting 'sidebar' is the following: purely potential flow past a liquid sphere would cause a dynamic pressure force that would distort the shape (from the $\boldsymbol{u} \cdot \nabla \boldsymbol{u}$ terms). Thus, when we speak of transients involving potential flow, they must be actually regarded as occurring at low Reynolds number (!), so that the dynamic pressure-relative to that caused by free-stream acceleration and added mass-is neglegibly small. This in turn seems to require, e.g., $u_{H}^{2} \ll<\dot{u}_{H} R$ in general一an inequality that is probably only 'sometimes' satisfied in the laboratory.

\section{The solid sphere}

Because the ultimate equation of motion that we seek is a really complicated one, it seems useful and appropriate to 'sneak-up' on it via a sequence of simpler problems. Thus, we begin with the ostensibly simple problem of applying $f=m a$ to a small, solid sphere in the linear (Stokes) flow regime. After discussing the additional features present when small nonlinearities are present ( $\operatorname{Re}>0$ but still 'small'), we shall move on to a simple drop and, finally, to the principal object of interest-the compound drop-whose treatment will be, unfortunately, incomplete and even somewhat speculative.

\section{Equation of motion for $\mathrm{Re}=\mathbf{0}$}

The (not-so-easy-to derive) equation of (vertical rectilinear) motion for a simple solid sphere in an unbounded quiescent and (very) viscous ( $\operatorname{Re}<<1$, for all time) fluid starting from rest is ( $z$ is upward, gravity downward)

$$
\begin{aligned}
& \frac{4}{3} \pi R^{3} \rho_{s} \dot{u}=f(t)+\frac{4}{3} \pi R^{3}\left(\rho-\rho_{s}\right) g-6 \pi R \mu\left[u(t)+R \int_{o}^{t} \frac{\dot{u}(\tau) d \tau}{\sqrt{\pi \nu(t-\tau)}}\right] \\
& +\int_{0}^{2 \pi} \int_{0}^{\pi}\left(-P_{H}^{A}(R, \theta) \cos \theta\right) R^{2} \sin \theta d \theta d \phi,
\end{aligned}
$$

where $\rho_{s}$ is the solid's density and $\rho$ the fluid density $(v=\mu / \rho$ is the kinematic viscosity), and $f(t)$ is an arbitrary z-directed 'body force' on the sphere (which we shall henceforth take to be zero). The total pressure force caused by the accelerations is easily 


$$
\begin{aligned}
& \text { evaluated from (86) with } \dot{u}_{H}=0, \dot{u}_{s}=\dot{u} \text {, and } \rho_{H}=\rho \text { to be [or use (82) } \\
& \left.R_{s} \rightarrow \infty, R_{c}=R, \dot{u}_{s}=0, \dot{u}_{c}=\dot{u} \text {, and } \rho_{s}=\rho\right]:-\frac{2}{3} \pi R^{3} \rho \dot{u} \text {, to give } \\
& \frac{4}{3} \pi R^{3}\left(\rho_{s}+\frac{1}{2} \rho\right) \dot{u}=\frac{4}{3} \pi R^{3}\left(\rho-\rho_{s}\right) g-6 \pi R \mu\left[u(t)+R \int_{o}^{t} \frac{i(\tau) d \tau}{\sqrt{\pi v(t-\tau)}}\right],
\end{aligned}
$$

with

where the term "added mass" now makes more sense; the force on the sphere caused by its acceleration relative to the fluid must also accelerate some of the surrounding fluid, with the net effect looking like the sphere itself has a larger 'effective' mass - in this case (a sphere) by one-half the mass of the displaced fluid.

Finally we come to the most difficult term - the integral term. Also called the history term or the Basset term for one of the early investigators (but see also Michaelides 1997-for alternate attributions; e.g., Boussinesq beat Basset), it properly accounts for the use of a steady Stokes drag term, $6 \pi R \mu u(t)$, during a transient situation-and we note that the recent past is more heavily weighted. It accounts for the time-dependent viscous diffusion of momentum from the sphere to the fluid — owing to the additional friction in the 'boundary layer'.

It is of some interest to examine the initial acceleration for this 'free-fall' problem:

$$
\dot{u}(0)=\frac{\rho-\rho_{s}}{\rho_{s}+\frac{1}{2} \rho} g,
$$

which is a potential flow result that correctly describes a viscous problem.

It is also interesting-although digressionary-to derive, from (89), the implied ordinary differential equation (ODE) that one might intuitively expect from applying $f=m \dot{u}$, rather than the $\mathrm{IDE}$ (integro-differential equation) that is (89). The derivation is non-trivial and involves applying Abel's theorem-see e.g., Basset (1910) for details. Here we present only the final result:

$$
\begin{aligned}
& \frac{\left[\frac{4}{3} \pi R^{3}\left(\rho_{s}+\frac{1}{2} \rho\right)\right]^{2}}{6 \pi R \mu} \ddot{u}+2\left[\frac{4 \pi}{3} R^{3}\left(\rho_{s}+\frac{1}{2} \rho\right)-3 \pi R^{3} \rho\right] \dot{u}+6 \pi R \mu u= \\
& \frac{4}{3} \pi R^{3}\left(\rho-\rho_{s}\right)+\frac{R}{\sqrt{\pi} v t}\left[6 \pi R \mu u(0)-\frac{4}{3} \pi R^{3}\left(\rho-\rho_{s}\right) g\right],
\end{aligned}
$$


about which the first thing to notice is that the $1 / \sqrt{t}$ singularity is only absent if $u(0)=u_{T}^{s}=\frac{2}{9} \frac{g R^{2}(\rho-\rho)}{\mu} g$ which is a trivial case, with solution $u(t)=u(0)=u_{T}^{s}$ ! The next thing that is apparent from (91) is the existence of 'negative damping'-i.e., instability -if the coefficient of the damping term, $\dot{u}(t)$ becomes negative. I.e., the 2 ndorder ODE above admits an unstable (exponentially growing) root if $\frac{4}{3}\left(\rho_{s}+\frac{1}{2} \rho\right)<3 \rho$,

or

$$
\rho_{s}<\frac{7}{4} \rho
$$

which tells us (at least) that we had better not try to integrate (91) numerically for the situation of most interest: $\rho \approx \rho_{s}$. We must attack (89) for a sphere that is too 'light'. Why can Newton's second law generate an unstable ODE? The brief answer is that it cannot; the unstable root is a spurious root that was introduced by raising the order of the equation. For more details on this issue, see Gresho et al. 1998. And this brings us to the final point regarding (91); it is not very difficult to find an analytical solution—which is

$$
u(t)=u_{T}^{s}\left\{1+\left[\frac{\frac{9}{2} \rho}{\left(\rho_{s}+\frac{1}{2} \rho\right)}\right]^{2}\left[\frac{\frac{1}{\sqrt{\lambda_{1}} e^{\lambda_{1} v t / R^{2}}} e r f c \sqrt{\lambda_{1} v t / R^{2}}-\frac{1}{\sqrt{\lambda_{2}}} e^{\lambda_{2} v t / R^{2}} e r f c \sqrt{\lambda_{2} v t / R^{2}}}{\lambda_{1}-\lambda_{2}}\right]\right\},
$$

which satisfies $u(0)=0$ and (90) and is stable (!) for all values of $\rho$ and $\rho_{s}$ where

$$
\lambda_{1}, \lambda_{2}=\frac{\frac{9}{4} \rho}{\left(\rho_{s}+\frac{1}{2} \rho\right)^{2}}\left[\frac{7}{2} \rho-2 \rho_{s} \pm \sqrt{\frac{9}{2} \rho\left(\frac{5}{2} \rho-4 \rho_{s}\right)}\right],
$$

the $+\operatorname{sign}$ taken for $\lambda_{1},-\operatorname{sign}$ for $\lambda_{2}$. Even though these $\lambda^{\prime} s$ may be real or complex with the real part positive or negative, it turns out that (93) is well-behaved (stable) for all values of $\rho$ and $\rho_{s}$, and always $u(t) \rightarrow u_{T}^{s}$ as $t \rightarrow \infty$. The rate that $u(t)$ approaches $u_{T}^{s}$ is, however, not as simple as one might first imagine. Both added mass and the history integral have a significant effect on the transient. If the history integral is neglected in (89), the solution is simple exponential, $e^{-t / \tau}$, with time constant

$$
\tau_{H}=\frac{2}{9} \frac{R^{2}\left(\rho_{s}+\frac{1}{2} \rho\right)}{\mu}
$$

whereas if both history and added mass are neglected (the naive approach to the sphere's. equation of motion), (89), gives 


$$
\tau_{H A}=\frac{2}{9} \frac{R^{2} \rho_{s}}{\mu}
$$

Thus, if $\rho \ll \rho_{s}$, (the fluid is a gas, for example) the simplest equation is a good approximation. Neither approximation is very accurate, however if $\rho \approx \rho_{s}$ or $\rho \ll \rho_{s}$, the first of which is our case, and samples of which can be seen in, e.g., Hjelmfelt and Mockros (1967), Mockros and Lai and (1969), and Clift et al. (1978). Both added mass and the history term are retarding in their effect on $u(t)$, with, usually, the history term being the more important. In all cases, however, we must have $\operatorname{Re}\left(u_{T}^{s}\right)<O(1)$, in order that the linear equations apply - although the added mass effects are present for any Reynolds number.

Before moving on to the fluid drop, it is worthwhile to generalize (89) for the case of a (still unidirectional, vertical) prescribed time-varying fluid velocity, say $u_{H}(t)$. This requires the use of (86), with $\rho_{\mathrm{H}}=\rho, R_{s}=R$, and $\dot{u}_{s}=\dot{u}$. It also uses relative velocity in the viscous term, and it is

$$
\begin{aligned}
\frac{4}{3} \pi R^{3} \rho_{s} \dot{u} & =\frac{4}{3} \pi R^{3}\left(\rho-\rho_{s}\right) g-6 \pi R \mu\left[u-u_{H}+R \cdot \int_{0}^{t} \frac{\dot{u}(\tau)-\dot{u}_{H}(\tau)}{\sqrt{\pi v(t-\tau)}} d \tau\right] \\
& +\frac{4}{3} \pi R^{3} \rho\left[\dot{u}_{H}+\frac{1}{2}\left(\dot{u}_{H}-\dot{u}\right)\right]
\end{aligned}
$$

where we have placed all of the potential flow/acceleration forces on the RHS, for clarity (the $\dot{u}$ term, of course, can be moved to the LHS to recover the added mass effect); viz., the new forcing term owing to acceleration of the host fluid consists of two parts: (i) the $\dot{u}_{H}$ term from the free-stream/solid body acceleration, per (86) and the discussion (remarks) below it, and (ii) the $\frac{1}{2} \dot{u}_{H}$ term from the added mass effect-for a total of $\frac{3}{2} \dot{u}_{H}\left(\times \frac{4}{3} \pi R^{3} \rho\right)$ as the total driving force from the host fluid. Added mass, a purely inviscid phenomenon that is also present for viscous flows, always involves a difference between two accelerations. To summarize these effects:

(1) The term $m \dot{u}_{H}\left(m=\right.$ fluid mass) is simply the effect of the $P=-\rho z \dot{u}_{H}$ force on the sphere from the 'free-stream' acceleration; a 'global' pressure field whose gradient is spatially uniform.

(2) The additional $\frac{1}{2} m \dot{u}_{H}$ is caused by the additional pressure force which itself is caused by the additional acceleration of the fluid in the neighborhood of the sphere (not uniform in space). 
(3) $\frac{1}{2}$ mi is truly the added mass: the inertia effect on $u$ of accelerating the fluid mass in the neighborhood of the sphere, which, of course, is still present even if $\dot{u}_{H}=0$.

(4) If $\iota_{H}$ is varying in space as well as time, additional effects (Faxen correction terms) enter; see below.

\section{Nonlinear $(\operatorname{Re}>0)$, effects, nonuniform far-field flow}

These additional 'real-world' complications are really that-so much so, in fact, that our discussion will be quite brief, referring to the extant literature and summarizing some brief-but-relevant portions of some of it, beginning with an important paper by Maxey and Riley (1983), (see too Maxey 1993) in which they corrected previous published errors and provided a correct equation of motion for a spherical particle in a non-uniform flow (in time and space) that is, however, still restricted to the linear (Stokes) regime. The most important addition to our Equation (89), is, besides the extension to 3-D, the effects of non-zero curvature ( $\nabla^{2} u$, basically) in the fluid flow field. This leads to the so-called Faxen correction terms (see too Kim and Karrila 1991) that must be added to three terms: the added mass term, the steady Stokes drag term, and the history integral. See the references for details.

For Re $>$ 0, Lovalenti and Brady (1993), have extended these results to small, but finite Reynolds number ( $\operatorname{Re}<1$ ), and to shapes other than spherical. Among many, one of their key conclusions of interest herein is that nonlinear effects hasten the approach to steady-state-especially on the longer time scale $\left[t>O\left(R^{2} / v\right)\right.$, the diffusional timescale]-owing to advection in the wake vis-à-vis radial diffusion. For small times, the transient Stokes solution is acceptably accurate. Thus, Equation (89), is probably pretty acceptable for most of our purposes-when $\operatorname{Re}<1$.

\section{The simple drop in Stokes flow}

The simple drop is actually quite far from 'simple' in the time-dependent setting. In fact, there are no closed form solutions available-even for the simplest case of release from rest in a quiescent fluid, via gravity. There is even (again-as for the easier case of a solid particle) a history of erroneous analyses, oversights, and-eventually-corrected versions. Thus, Sy and Lightfoot (1971) made the first attempt at a transient analysis of a simplc drop-but, because of an oversight during nondimensionalization, their results are only valid when the two fluids have the same kinematic viscosities, which * $\Rightarrow \mu_{i} / \rho_{i}=\mu_{o} / \rho_{o}$. The 'problem' with a 2-fluid system, and the problem not solved by 
these authors, is that the 2 fluids will generally display 2 different 'spinup' rates because of the generally disparate diffusional time scales $\tau_{i} \equiv v_{i} / R^{2}, \tau_{o} \equiv v_{o} / R^{2}$. (See Michaelides (1997) for further discussion of historical 'errors', and their corrections.) Chisnell (1987) recognized this issue, and corrected it-at least in the Laplace transform domain. But, in contrast to the solid sphere case, it is (ostensibly) not possible to find an analytic inverse and thus a closed-form solution in the time domain. Hence, the 'global' equation of motion for the spherical drop is, unfortunately, not available in any simple form. Another of Chisnell's results is more directly useful, however: he showed, as we did above for the steady state case (drop falling at terminal speed), that the transient Stokes case also does not 'upset' the normal force balance on the surface, with the desirable result (again) being that a spherical drop remains spherical for all time (for $\mathrm{Re}=0$ ). This result is consistent with one by Koh and Leal (1989), in which they performed a stability analysis of the steady state translating droplet (at $u_{T}$ ) by perturbing its shape (to prolate or oblate spheroids) and determining if it returns to spherical. For small perturbations, the shapes did return to spherical-which is consistent with Chisnell's transient analysis.

The closest thing available for the global equation of motion of a 'falling' drop is given in Lovalenti and Brady (1993) as a formal (integral) solution. (See also Michaelides 1997.) Here we present our version of Newton's second law for a translating (at $u_{i}$ ) drop in a moving fluid (at $u_{o}$ ) that is only 'mostly correct', but still (we believe) somewhat useful-as will be its generalization to the compound droplet:

$$
\begin{aligned}
\frac{4}{3} \pi R^{3} \rho_{i} \dot{u}_{i} & =\frac{4}{3} \pi R^{3}\left(\rho_{o}-\rho_{i}\right) g-6 \pi \mu_{o} R \cdot \frac{2 / 3+\mu_{i} / \mu_{o}}{1+\mu_{i} / \mu_{o}}\left[u_{i}-u_{o}+R \int_{o}^{t} \frac{\dot{u}_{i}(\tau)-\dot{u}_{o}(\tau)}{\sqrt{\pi v_{o}(t-\tau)}} d \tau\right] \\
& +\frac{4}{3} \pi R^{3} \rho_{o}\left[\dot{u}_{o}+\frac{1}{2}\left(\dot{u}_{o}-\dot{u}_{i}\right)\right]+c(t)
\end{aligned}
$$

wherc $c(t)$ is a 'correction' term (not explicitly known) that includes a new history integral and is hopefully 'small' in some sense. We have encouragement in this "hope" from Lawrence and Weinbaum (1986), who analyzed the equation of motion of solid spheroids and concluded that the correction term is "...in most cases ... very small." Also, in Yang and Leal (1991) who 'introduced' the new memory integral term for the liquid drop and showed a modified 'Basset' history integral term, (albeit with a similar nondimensionalization error that negates some of their results), is stated, "It is, however, worth commenting that the qualitative dependence of the total memory function, modified Basset plus new term, is very similar to the (original) Basset memory term for the entire. 
range of $t$...". Finally, as this equation is merely a 'stepping-stone' to those of the next section, we now drop the simple drop (splat!).

\section{E. The compound drop in Stokes flow}

Speculation and approximations necessarily increase as we move closer to our final objective- - to determine the fluid dynamical behavior of a core and its shell (both assumed spherical) as they move about (albeit unidirectionally) in the host fluid. We in general must consider 3 different densities and 3 different viscosities. Mass transfer-in the form of solvent loss by diffusion from the shell to the host, which also affects the fluid properties, including surface tension, should be accounted for as well. The changing rheology of the shell, including non-Newtonian behavior, should also be included.

But we are not yet smart enough to accurately describe-or even to model-the appropriate physics, and physical chemistry. Thus, we shall continue to assume constant property Newtonian behavior in the Stokes flow regime. Even this modest and simplified approach can only be approximately described, unfortunately.

\section{Equations of motion}

Building upon what is known about simpler systems, and guessing a bit about what is not known, we put forth the following approximate equations of motion for core and shell, beginning with the rather stringent assumption that the two are concentric and thus, actually only apply 'instantaneously'-unless they never stray far from concentricity. Also required is a simplified initial condition: $u_{s}=u_{c}=u_{H}(0)$ to avoid the complicating introduction of Dirac delta functions and related issues. These restrictions, however, will not preclude our learning some things about the transient response of such droplets.

\section{Core equation of motion:}

$$
\begin{aligned}
& \frac{4}{3} \pi R_{c}^{3} \rho_{c} \dot{u}_{c} \cong \frac{4}{3} \pi R_{c}^{3}\left(\rho_{s}-\rho_{c}\right) g-6 \pi \mu_{s} R_{c} K_{c s} \frac{2 / 3+\mu_{c} / \mu_{s}}{1+\mu_{c} / \mu_{s}}\left[u_{c}-u_{s}+\right. \\
& \left.R_{c} \int_{0}^{t} \frac{\dot{u}_{c}(\tau)-\dot{u}_{s}(\tau)}{\sqrt{\pi v_{s}(t-\tau)}} d \tau\right]-6 \pi \mu_{H} R_{c} K_{c H}\left[u_{c}-u_{H}+R_{c} \int_{0}^{t} \frac{\dot{u}_{c}(\tau)-\dot{u}_{H}(\tau)}{\sqrt{\pi v_{c}(t-\tau)}} d \tau\right] \\
& +\frac{4}{3} \pi R_{c}^{3} \rho_{s}\left[\frac{\dot{u}_{s} R_{s}^{3}-\dot{u}_{c} R_{c}^{3}+\frac{1}{2} R_{s}^{3}\left(\dot{u}_{s}-\dot{u}_{c}\right)}{R_{s}^{3}-R_{c}^{3}}\right]
\end{aligned}
$$

which merits a few explanatory remarks: 
1. $K_{c s}$ is a (steady Stokes) factor $(\geq 1)$ that attempts to account for the fact that the shell fluid is not unbounded. It is a "wall correction factor" that, for a rigid (no slip) surface at the shell's outer radius, looks like that shown in Fig. 7 and in Equation (99) below, from Happel and Brenner (1965), and is a strong function of radius ratio and a weak function of viscosity ratio. While $K_{c s}$ is not readily available for the liquid-liquid interface, although it is given implicitly in Sadhal and Oguz (1985) for the more general (eccentric) case, we will assume that it is not too different from the known result for a rigid boundary $\left(\lambda=\mu_{c} / \mu_{s}\right.$, as before, and $\left.r \equiv R_{c} / R_{s}\right)$ :

$$
K_{c s}=\frac{1-\left(\frac{\lambda-1}{\lambda+\frac{2}{3}}\right) r^{5}}{1-\frac{9}{4}\left(\frac{\lambda+2 / 3}{\lambda+1}\right) r+\frac{5}{2}\left(\frac{\lambda}{1+\lambda}\right) r^{3}-\frac{9}{4}\left(\frac{\lambda-2 / 3}{\lambda+1}\right) r^{5}+\left(\frac{\lambda-1}{\lambda+1}\right) r^{6}},
$$

showing a very strong viscous effect for thin-shelled drops-another part of the answer to the question, "Why does the core stay in the shell?" Note that experimental results also support the contention that thin walls favor robustions/stability (Hamilton (1997-p.6). In fact, for $R_{c} \rightarrow R_{s} \equiv R$ and $R_{s}-R_{c} \equiv \delta R$, the $r \rightarrow 1$ limit of (99) is

$$
K_{c s} \cdot \frac{2 / 3+\lambda}{1+\lambda} \cong \frac{1}{3}\left(\frac{R}{\delta R}\right)^{3}
$$

2. $K_{c H}$, in the 'friction' term between core and host, is available in Brunn and Roden (1985), also for the steady state case. It is, with $r \equiv R_{c} / R_{s}$ and $\mu_{i j} \equiv \mu_{i} / \mu_{j}$,

$$
\begin{gathered}
K_{c H}=\frac{2}{3}\left(\frac{r}{1-r}\right) . \\
\frac{2 \mu_{s c}+(1-r)\left(3+6 r+4 r^{2}+2 r^{3}\right) f(r)}{4 \mu_{s c} \mu_{s H}+2 \mu_{s c}\left(1+\mu_{c H}\right)\left(1-r^{2}\right)\left(2+r+2 r^{2}\right) f(r)+(1-r)^{2}\left(4+7 r+4 r^{2}\right) f(r)},
\end{gathered}
$$

where $f(r) \equiv 1 /\left(1+r+r^{2}+r^{3}+r^{4}\right)$. Note that $K_{c H}$ also becomes unbounded, like $O[1 /(1-r)]$, for $R_{c} \rightarrow R_{s}$; thin shells cause large forces and, as mentioned earlier, these tend to reduce the flow toward zero in both core and shell and keep the core inside the shell.

3. The last term, of course, is from the potential flow analysis-see (82) and (96)-and includes the effects of free stream acceleration and, in the difference' term, added mass. 
4. Omitted completely is the analogous so-called "correction term" for the simple drop, per (97), on the assumption that it is not too important.

\section{Shell equation of motion:}

$$
\begin{aligned}
& \frac{4}{3} \pi\left(R_{s}^{3}-R_{c}^{3}\right) \rho_{s} \dot{u}_{s} \cong \frac{4}{3} \pi\left[\rho_{H} R_{s}^{3}-\rho_{s}\left(R_{s}^{3}-R_{c}^{3}\right)-\rho_{c} R_{c}^{3}\right] g+6 \pi \mu_{s} R_{c} K_{c s} \cdot \frac{2 / 3+\mu_{c} / \mu_{s}}{1+\mu_{c} / \mu_{s}} \cdot[ \\
& \left.u_{c}-u_{s}+R_{c} \int_{o}^{t} \frac{\dot{u}_{c}(\tau)-\dot{u}_{s}(\tau)}{\sqrt{\pi v_{s}(t-\tau)}} d \tau\right]-6 \pi \mu_{H} R_{s} K_{s H} \cdot \frac{2 / 3+\mu_{s} / \mu_{H}}{1+\mu_{s} / \mu_{H}} \cdot\left[u_{s}-u_{H}+R_{s} \int_{o}^{t} \frac{\dot{u}_{s}(\tau)-\dot{u}_{H}(\tau)}{\sqrt{\pi v_{H}(t-\tau)}} d \tau\right] \\
& +\frac{4}{3} \pi R_{s}^{3} \rho_{H}\left[\dot{u}_{H}+\frac{1}{2}\left(\dot{u}_{H}-\dot{u}_{s}\right)\right]-\frac{4}{3} \pi R_{c}^{3} \rho_{c} \dot{u}_{c}
\end{aligned}
$$

which clearly requires more remarks:

1. In both the buoyancy term (buoyancy minus weight, actually) and the potential flow (acceleration) term, the signs of the core fluid forces on the shell are opposite those of the host fluid forces. In the former, it causes a downward 'buoyancy' force and in the latter a 'reaction'-force to the free-stream acceleration of the core-see (84) and (81), wherein now the pressure force at $r=R_{c}$ is $+P_{s}^{A}\left(R_{c}, \theta\right) \cos \theta$.

2. The other potential flow force (free-stream plus added mass) from the host comes from (86).

3. The $K_{c s}$ term acts on the shell with opposite sign to that for the core.

4. The friction factor in the term between shell and host, $K_{s H}$, is available for steady concentric spheres in Sadhal Oguz (1985) (implicitly), Brunn and Roden (1985), and, more explicitly in Rushton and Davies (1983), whose result we shall use $\left(r \equiv R_{c} / R_{s}\right.$ and $\mu_{s H} \equiv \mu_{s} / \mu_{H}$, still $):$

$$
f_{s H} \equiv K_{s H} \cdot \frac{2 / 3+\mu_{s H}}{1+\mu_{s H}}=\frac{2}{3} \cdot \frac{\mu_{c H}+6 \mu_{s H}^{2} \cdot G(r)+\mu_{s H} \cdot\left(2+3 \mu_{c H}\right) \cdot F(r)}{\mu_{c H}+4 \mu_{s H}^{2} \cdot G(r)+2 \mu_{s H}\left(1+\mu_{c H}\right) \cdot F(r)},
$$

where

$$
F(r) \equiv \frac{(1+r)\left(2 r^{2}+r+2\right)}{(1-r)\left(4 r^{2}+7 r+4\right)}
$$

and

$$
G(r) \equiv \frac{1-r^{5}}{(1-r)^{3}\left(4 r^{2}+7 r+4\right)}
$$


The function $f_{s H}$ properly tends to the simple drop limit, $f_{s H}=\frac{2 / 3+\mu_{s H}}{1+\mu_{s H}}$ for $r \rightarrow 0$ and to $f_{s H} \rightarrow 1$ for $r \rightarrow 1$, the rigid sphere case (because the flow in core and shell is then zero).

One of the immediate uses of the core and shell equations of motion is to estimate the initial accelerations $\left(\dot{u}_{c}^{0}\right.$ and $\dot{u}_{s}^{0}$ ) and time constants of the system. Thus, omitting the history integrals in (98) and (102) and transposing the 'added mass' terms to the left sides yields

$$
\begin{aligned}
& \frac{4}{3} \pi R_{c}^{3}\left[\rho_{c}+\rho_{s} \cdot \frac{R_{c}^{3}+\frac{1}{2} R_{s}^{3}}{R_{s}^{3}-R_{c}^{3}}\right] \dot{u}_{c}^{0} \cong \frac{4}{3} \pi R_{c}^{3}\left(\rho_{s}-\rho_{c}\right) g-6 \pi \mu_{s} R_{c} K_{c s} \cdot \frac{2 / 3+\mu_{c} / \mu_{s}}{1+\mu_{c} / \mu_{s}} \\
& \left(u_{c}^{0}-u_{s}^{0}\right)-6 \pi \mu_{H} R_{c} K_{c H}\left(u_{c}^{0}-u_{H}^{0}\right)+2 \pi R_{c}^{3} \rho_{s} \frac{R_{s}^{3}}{R_{s}^{3}-R_{c}^{3}} \dot{u}_{s}^{o}
\end{aligned}
$$

for the core, and

$$
\begin{aligned}
& \frac{4}{3} \pi\left[\left(R_{s}^{3}-R_{c}^{3}\right) \rho_{s}+\frac{1}{2} R_{s}^{3} \rho_{H}\right] \dot{u}_{s}^{o} \cong \frac{4}{3} \pi\left[\rho_{H} R_{s}^{3}-\rho_{s}\left(R_{s}^{3}-R_{c}^{3}\right)-\rho_{c} R_{c}^{3}\right] g+6 \pi \mu_{s} R_{c} K_{c s} \\
& \left(\frac{2 / 3+\mu_{c} / \mu_{s}}{1+\mu_{c} / \mu_{s}}\right) \cdot\left(u_{c}^{o}-u_{s}^{o}\right)-6 \pi \mu_{H} R_{s} K_{s H} \cdot \frac{2 / 3+\mu_{s} / \mu_{H}}{1+\mu_{s} / \mu_{H}}\left(u_{s}^{o}-u_{H}^{o}\right)+2 \pi R_{s}^{3} \rho_{H} \dot{u}_{H}^{o}-\frac{4}{3} \pi R_{c}^{3} \rho_{c} \dot{u}_{c}^{o}
\end{aligned}
$$

for the shell. Noteworthy for $R_{c} \rightarrow R_{s}$ is the powerful influence of the shell on the core; i.e., (104) then becomes $\dot{u}_{c}^{o} \cong \dot{u}_{s}^{o}$-the core follows the shell, a purely invisid (potential flow) effect.

Setting the generally unknown $\dot{u}_{H}^{o}$ to zero and solving for the two initial accelerations with $u_{s}^{o}=u_{c}^{o}=u_{H}^{o}$ yields

$$
\dot{u}_{c}^{o}=\frac{\left[\left(R_{s}^{3}-R_{c}^{3}\right) \rho_{s}+\frac{1}{2} R_{s}^{3} \rho_{H}\right] \cdot\left(\rho_{s}-\rho_{c}\right)+\frac{3}{2} \rho_{s}\left[R_{s}^{3} \rho_{H}-\left(R_{s}^{3}-R_{c}^{3}\right) \rho_{s}-R_{c}^{3} \rho_{c}\right]}{\left[\rho_{c}+\rho_{s} \cdot \frac{R_{c}^{3}+\frac{1}{2} R_{s}^{3}}{R_{s}^{3}-R_{c}^{3}}\right] \cdot\left[\left(R_{s}^{3}-R_{c}^{3}\right) \rho_{s}+\frac{1}{2} R_{s}^{3} \rho_{H}\right]+\frac{3}{2} \frac{R_{c}^{3} R_{s}^{3}}{R_{s}^{3}-R_{c}^{3}} \rho_{s} \rho_{c}} g
$$

and

$$
\dot{u}_{s}^{o}=\frac{\left[\rho_{c}+\rho_{s} \cdot \frac{R_{c}^{3}+\frac{1}{2} R_{s}^{3}}{R_{s}^{3}-R_{c}^{3}}\right] \cdot\left[R_{s}^{3} \rho_{H}-\left(R_{s}^{3}-R_{c}^{3}\right) \rho_{s}-R_{c}^{3} \rho_{c}\right]-R_{c}^{3} \rho_{c}\left(\rho_{s}-\rho_{c}\right)}{\left[\rho_{c}+\rho_{s} \cdot \frac{R_{c}^{3}+\frac{1}{2} R_{s}^{3}}{R_{s}^{3}-R_{c}^{3}}\right] \cdot\left[\left(R_{s}^{3}-R_{c}^{3}\right) \rho_{s}+\frac{1}{2} R_{s}^{3} \rho_{H}\right]+\frac{3}{2} \frac{R_{c}^{3} R_{s}^{3}}{R_{s}^{3}-R_{c}^{3}} \rho_{s} \rho_{c}} g
$$

where we point out that the appropriate limiting case for both core and shell,$\dot{u}_{o} \rightarrow\left(\rho_{H}-\rho_{c}\right) g /\left(\rho_{c}+\frac{1}{2} \rho_{H}\right)$ for $R_{c} \rightarrow R_{s}$, per $(90)$, is recovered. 
Finally, omitting the driving forces and seeking solutions to (102) and (104) of the form $u \sim e^{-t / \tau}$ yields the two (initial) time constants;

$$
\lambda_{ \pm}=1 / \tau_{ \pm}=\frac{-b \pm \sqrt{b^{2}-4 a c}}{2 a},
$$

where

$$
a \equiv m_{c} m_{s}+m_{s c} m_{c s},
$$

$$
b \equiv-\left[m_{c} k_{s}+m_{s} k_{c}+\left(m_{c s}-m_{s c}\right) k_{c s}\right] \text {, }
$$

and

$$
c=k_{c} k_{s}-k_{c s}^{2}
$$

where

$$
\begin{gathered}
m_{c} \equiv \frac{4}{3} \pi R_{c}^{3}\left[\rho_{c}+\rho_{s} \cdot \frac{R_{c}^{3}+\frac{1}{2} R_{s}^{3}}{R_{s}^{3}-R_{c}^{3}}\right], \\
m_{s} \equiv \frac{4}{3} \pi\left[\left(R_{s}^{3}-R_{c}^{3}\right) \rho_{s}+\frac{1}{2} R_{s}^{3} \rho_{H}\right], \\
m_{s c} \equiv 2 \pi R_{c}^{3} \rho_{s} \frac{R_{s}^{3}}{R_{s}^{3}-R_{c}^{3}}, \\
m_{c s} \equiv \frac{4}{3} \pi R_{c}^{3} \rho_{c}, \\
k_{c} \equiv k_{c s}+k_{c H}, \\
k_{s} \equiv k_{c s}+k_{s H},
\end{gathered}
$$

where

$$
\begin{gathered}
k_{c s} \equiv 6 \pi \mu_{s} R_{c} K_{c s} \frac{2 / 3+\mu_{c} / \mu_{s}}{1+\mu_{c} / \mu_{s}}, \\
k_{c H}=6 \pi \mu_{H} R_{c} K_{c H}, \\
k_{s H} \equiv 6 \pi \mu_{H} R_{s} K_{s H} \frac{2 / 3+\mu_{s} / \mu_{H}}{1+\mu_{s} / \mu_{H}} .
\end{gathered}
$$

and

Since it will turn out that $k_{c H} \ll k_{c s}$ and $k_{s H} \ll k_{c s}$ because the core-shell coupling is very strong, it is of some interest to present and examine more closely the full ODE solution to (104) and (105) under these conditions-which of course neglects two important points: (1) the history terms will reduce the response rates and (2) the core will generally not remain centered. Thus, for small $k_{c H}$ and $k_{s H}$ with $u_{c}(0)=u_{c}^{o}$ and $u_{s}(0)=u_{s}^{o}$ given, $u_{H}$ given (and constant) and $g=0$ (for simplicity), 


$$
\begin{aligned}
& u_{s}(t) \cong u_{H}\left(1-e^{-\lambda_{-} t}\right)+\frac{\left(m_{c}+m_{c s}\right) u_{c}^{o}+\left(m_{s}-m_{s c}\right) u_{s}^{o}}{m_{c}+m_{c s}+m_{s}-m_{s c}} e^{-\lambda_{-} t}+\frac{\left(m_{s}-m_{s c}\right)\left(u_{c}^{o}-u_{s}^{o}\right)}{m_{c}+m_{c s}+m_{s}-m_{s c}} e^{-\lambda_{+} t}
\end{aligned}
$$

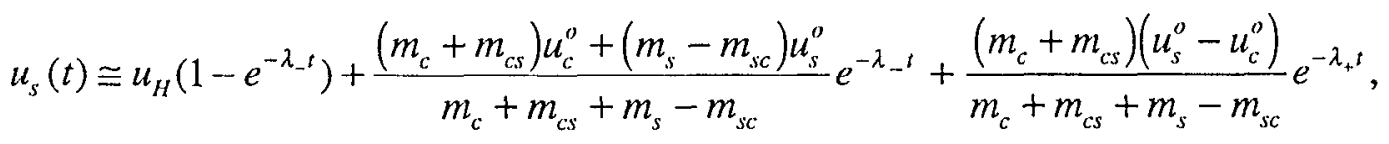

$$
\begin{aligned}
& \text { where } \\
& \lambda_{+} \cong k_{c s} \cdot \frac{m_{c}+m_{c s}+m_{s}-m_{s c}}{m_{c} m_{s}+m_{s c} m_{c s}} \\
& \text { and } \\
& \lambda_{-} \cong \frac{k_{c H}+k_{s H}}{m_{c}+m_{c s}+m_{s}-m_{s c}},
\end{aligned}
$$

about which the following remarks are relevant:

1. $\lambda_{+}$is the 'fast' transient because $k_{c s}$ is much larger than $k_{c H}$ and $k_{s H}$. Ergo, $\lambda_{-}$is the slow transient $\left(\lambda_{-} \ll \lambda_{+}\right)$.

2. If $u_{c}^{o}=u_{s}^{o}=u_{o}$, the solution for both core and shell is simply $u(t) \cong u_{o} e^{-\lambda_{-} t}+u_{H}\left(1-e^{-\lambda_{-} t}\right)$.

3. The fast transient causes a rapid equilibration to the long-term, slowly-decaying solution,

$$
\begin{aligned}
& u_{c}(t) \approx u_{s}(t) \approx u_{e q} e^{-\lambda_{-} t}+u_{H}\left(1-e^{-\lambda_{-} t}\right), \text { where } u_{e q} \text { is the 'equilibrium' velocity, } \\
& u_{e q}=\frac{\left(m_{c}+m_{c s}\right) u_{c}^{o}+\left(m_{s}-m_{s c}\right) u_{s}^{o}}{m_{c}+m_{c s}+m_{s}-m_{s c}}
\end{aligned}
$$

4. The main point of all this is this: the core follows the shell as it is moved about by the host fluid.

We will attempt to utilize these and earlier results later. Now we move to the last 'theoretical' issue to be addressed in this paper.

\section{Core centering}

One of the seemingly-omnipresent and very fortunate aspects of the dynamics of compound drops that is, unfortunately, not yet fully understood theoretically, is the following: 'excitation' of the shell, in a variety of manners, including: stirring the host fluid, heating the host fluid, tumbling the compound droplet via motion of the host fluid, acoustic oscillations, or even impulsive loading and perhaps simple gravitational settling, all tend to cause the core to become centered-or close to centered-in the shell. This piece 
of 'good news' is, of course, one of the 'drivers' of the microencapsulation route to making spherical targets.

Whereas we shall later add our own contribution to the core-centering phenomenon, in this section, we shall describe what has been observed and, partially, what has been done to help explain the observations.

Saffren et al. 1982, who performed experiments in a neutral density tank with water as the shell fluid and silicone oil as core and host, state: "Though this centering phenomenon takes place within a few cycles of oscillation (at the mode 2 frequency), the centering force seems to depend, among other things, on the oscillation amplitude and the shell thickness."

Using acoustic levitation of a compound drop in air, Lee et al. (1982) found that the centering effect is much stronger for small (sub-millimeter) than large (several $\mathrm{mm}$ ) drops. "When the frequency of the amplitude modulation is tuned to the natural oscillation frequency of the compound droplet system, a large amplitude oscillation of the liquid shell is produced driving the core to the center of the system in a fraction of a second." And, referring to this study, Kendall (1986) states: "In another oscillation study involving substantial density mismatch, the forced oscillation of an acoustically levitated, lightweight hollow glass shell $(\rho \cong 5)$ thickly coated with a low-viscosity liquid $\left(\mathrm{H}_{2} \mathrm{O}\right)$, induced centering, showing that the forces attainable by means of oscillation are very strong".

A nice summary of experimental results is provided by Lee and Wang (1988): "Many experiments have indicated a tendency for a compound drop in oscillation to become concentric. In a zero-gravity experiment (using a NASA KC-135 aircraft), water droplets with injected air bubbles consistently assumed concentric spherical form (T.G. Wang and D.D. Elleman 1981, private communication). Free-falling hollow liquid shells, about $1 \mathrm{~mm}$ in radius, produced by annular jet instability, tended to remain concentric. In an acoustic levitation experiment, the hollow shell produced by introduction of air into a $1 \mathrm{~mm}$ drop was found to resist breakup when oscillation was induced. It has also been demonstrated (Saffren et al. 1982) in a neutral buoyancy tank that a compound drop formed of immiscible liquids becomes concentric when excited into oscillations. A similar effect has been seen when a layer of liquid coating a small solid sphere in acoustic levitation was forced into a capillary oscillation." They then go on to generate an inviscid model for a liquid shell (gaseous core and host) that was later found not to agree well with experiments (see below). 
In some acoustic levitation experiments performed aboard the space shuttle Columbia, Wang et al. (1994) performed experiments with both liquid and gaseous cores, liquid shells, and a gaseous host. They concluded that, even for the simplest case of a liquid shell with gaseous neighbors, any purely inviscid theory was invalid - the system is highly damped. "The observation seems to suggest that as far as the nonlinear effect called centering is concerned, viscosity should be taken into account in order to obtain a realistic prediction."

Another paper that cited failure of purely inviscid models is that of Lee et al. (1996) - -again for the simple case of a gas bubble, a liquid shell and a gaseous host; "...Therefore, a free oscillation is not efficient in bringing about centering, because the centering force decays in time before centering is complete," and "Centering can occur only if the capillary oscillation is sustained by an external source, such as a modulated acoustic field."

Unfortunately, there is still not available a viscous analysis that has proven to be successful in explaining the physics of core-centering. The ad hoc model of Norimatsu-in this volume-is, however, a potentially-winning candidate, even though it is not derived from so-called 'first principles' (Navier-Stokes equations).

\section{Applications}

Finally, we will apply some of the above theory to the area of interest to 'target fabricators'. Realizing that our compound droplet is quite a moving target (before it becomes a final stationary target for the laser beams!) because of the significantly timevarying composition of the shell (in addition to droplet motion), and admitting that some of the physical properties are not very well known, we will base most of our calculations on a so-called nominal case in which the following description applies: $R_{c}=0.098 \mathrm{~cm}, R_{s}=$ $0.109 \mathrm{~cm}\left(R_{c} / R_{s} \cong 0.90\right)$. It should be noted that encapsulated droplets generally have an oil wall thickness of 150 to $250 \mu \mathrm{m}$ as initially formed, depending on the polymer concentration and desired final (solid) wall thickness. As the dilute polymer solution loses solvent, a final dry wall of 20 to $30 \mu \mathrm{m}$ is formed. Our particular choice of $R_{S}-R_{\mathcal{C}}$ $(110 \mu \mathrm{m})$ in these examples is meant to represent some 'midpoint' in the drying process for a NIF-scale shell, although it should be born in mind that $R_{C} / R_{S}$ actually could vary between $\sim 0.98$ to $\sim 0.77$. We will also use $R \cong 0.1 \mathrm{~cm}$ when dealing with a simple drop. The nominal properties are (cgs): 


\begin{tabular}{|c|c|c|c|}
\hline & Core & Shell & Host \\
\hline$\rho(g m / c c)$ & 1.00 & 1.01 & 1.00 \\
\hline$\mu($ poise $)$ & 0.01 & $0.1,1,10$ & 0.01 \\
\hline$\gamma($ dyne $/ \mathrm{cm})$ & & & \\
\hline
\end{tabular}

in which it is clear that the shell's viscosity is highly variable, and clearly depends upon the initial polymer concentration and degree of drying. The densities we have selected are at best approximate, but as has been pointed out the key dependencies are on the density differences across interfaces, and in this regard $0.01 \mathrm{~g} / \mathrm{cm}^{3}$ is a reasonable estimate. Note also that while we have used a value of 10 dynes/cm for the interfacial tension, the actual value will clearly depend upon the solution compositions and may range from $\approx 1$ to 30 or more.

\section{A. Static droplet in a density column}

Equation (20) gives the MooR for this case. Choosing an easily attainable valuc for $\beta$ of $0.01 \mathrm{~cm}^{-1}$, gives $M o o R \cong 0.025 \mu \mathrm{m}$; not a problem.

\section{B. Terminal velocity and loss of sphericity}

\section{Stokes flow}

The 'effective' droplet density $(\bar{\rho})$ for a simple drop is obtained from $\bar{\rho} R_{s}^{3}=\rho_{c} R_{c}^{3}+\rho_{s}\left(R_{s}^{3}-R_{c}^{3}\right)$, as $\bar{\rho} \equiv 1.0027$ (changing $\rho_{s}$ to 1.02 changes $\bar{\rho}$ to $\sim 1.0055$ ) and, from (43) and (53) we have

$$
\frac{2}{9} \frac{g R_{s}^{2}}{\mu_{H}}\left(\bar{\rho}-\rho_{H}\right)=u_{\text {solid }}<u_{\text {compound }}<u_{\text {simple }}=u_{\text {solid }} \times \frac{1+\mu_{s} / \mu_{H}}{\frac{2}{3}+\mu_{s} / \mu_{H}},
$$

in which the right-most equation assumes that the shell viscosity 'dominates', which is probably a good guess since its viscosity is large (say 1 poise). Thus,

$$
0.653 \mathrm{~cm} / \mathrm{sec}<u_{\text {compound }}<0.655 \mathrm{~cm} / \mathrm{sec} \text {, }
$$

giving a terminal Reynolds number of $\operatorname{Re}=u R / v_{H} \cong 6.5$, which, unfortunately, somewhat vitiates the assumption of Stokes flow. To have $\operatorname{Re}<1$ requires $u<0.1 \mathrm{~cm} / \mathrm{sec}^{-}$ 
which would require $\Delta \rho<\sim 0.0005$ which is hard to obtain. Thus, if terminal velocity is reached, we may be somewhat out of the range of Stokes flow.

\section{2. $\operatorname{Re}>0$ (Navier-Stokes flow)}

If we apply (58) to this nominal case, we get (for $\mu_{s}=1$ ) $u_{T} \cong 0.35$, giving $\mathrm{Re} \cong 3.5$ a little better, and probably more accurate.

For a final free-fall estimate, we recall that thin-shelled compound droplets behave more like solid spheres than simple drops. On this assumptions, we can get a good estimate of $u_{T}$ from some correlations given in Clift et al. (1978), as follows:

(1) Compute $N_{D}=C_{D} \operatorname{Re}_{T}^{2}=4 \rho \Delta \rho g D^{3} / 3 \mu^{2}$,

where $C_{D}$ is the drag coefficient and $D$ is the diameter. Inserting our parameters gives $N_{D} \cong 314$.

(2) A curve-fit equation that applies for this value of $N_{D}$ then gives $\operatorname{Re}_{T}$ :

$$
\begin{aligned}
\log _{10} \operatorname{Re}_{T} & =-1.7095+1.33438 \log _{10} N_{D}-0.11591\left(\log _{10} N_{D}\right)^{2} \\
& =0.899 ; \Rightarrow \operatorname{Re}_{T} \equiv \frac{2 R u_{T}}{v}=7.93 \Rightarrow
\end{aligned}
$$

$u_{T} \cong 0.40 \mathrm{~cm} / \mathrm{sec}$, agreeing quite well with our earlier estimate-even though the definition of Reynolds number is a factor of 2 different. (Most seem to use the diameter as the characteristic length.)

\section{Droplet shape}

Applying now (59) using $u=0.35 \mathrm{~cm} / \mathrm{sec}$ and admitting to a Reynolds number that is somewhat larger than it should be in order to believe (59), gives

$$
\text { MOOR }=3 \times .23 \times 1 \times .35^{2} \times .1^{2} / 10 \cong 0.8 \times 10^{-4} \mathrm{~cm}=0.8 \mu m,
$$

which is probably tolerable-but barely, and worse yet if $\gamma$ is smaller.

For what it's worth, we shall also use one of the results of Brunn and Roden (1985) to estimate the compound droplet's shape; in their Fig. 6 is shown a "typical" set of shape factors:

$$
\begin{aligned}
r_{s}(\theta) & =R_{s}\left[1-\epsilon_{s} P_{2}(\cos \theta)\right] \\
\text { and } r_{c}(\theta) & =R_{s}\left[1+\epsilon_{c} P_{2}(\cos \theta)\right]
\end{aligned}
$$


where $\epsilon_{s}$ and $\epsilon_{c}$ are very complicated functions of the problem parameters. For the "typical" set shown in their Fig. 6, we get, using $R_{c} / R_{s}=0.9, \in_{s} \cong 0.083 R_{s} \rho_{s} u_{s}^{2} / \gamma_{s H}$ and $\epsilon_{c} \cong 0.056 R_{c} \rho_{c} u_{c}^{2} / \gamma_{c s}$. For our nominal case, (and $u_{c}=u_{s}=0.35$ ) these give $\epsilon_{s} \cong 1.1 \times 10^{-4}, \epsilon_{c} \cong 6.7 \times 10^{-5}$, and thus

$$
\begin{aligned}
\operatorname{MooR}_{s} & =2\left[r_{s}(\pi / 2)-r_{s}(0)\right]=3 \in_{s} R_{s} \cong 0.36 \mu m \\
\text { and } \operatorname{MooR}_{c} & =2\left[r_{c}(0)-r_{c}(\pi / 2)\right]=3 \in_{c} R_{c} \cong 0.20 \mu m
\end{aligned}
$$

the latter being a prolate spheroid. It is reassuring, at least, to see reasonable agreement with the simple drop result from the Taylor-Acrivos theory; it tends to lend credence to each.

These results seem to say that freely-falling droplets (in a drop tower, for example) can probably be made to meet the specs-it depends mostly on the 'control' of $\Delta \rho$.

Taylor and Acrivos (1964) also give an equation for the effect on the drag force caused by shape deformation. It is, however, not of any importance for our purposes-being a "second-order" (or less) effect on droplet dynamics and terminal speed.

\section{Droplet shape in a linear shear flow}

Assuming a beaker whose contents are stirred at a frequency of 1 revolution per sec $(f=1 \mathrm{~Hz})$ gives a shear rate of $\mathrm{G}=2 \pi f$, which gives, using $(61), \operatorname{MooR}=4 \times .01 \times 2 \pi$ $\times(0.1)^{2} / 10=2.5 \times 10^{-4} \mathrm{~cm}=2.5 \mu \mathrm{m}$, which could be a problem.

\section{Capillary waves}

\section{Inviscid simple drop}

The mode 2 frequency from (69), is $\omega_{2}^{I} \cong 220 \sec ^{-1}$ for $R=0.1$; a $35 \mathrm{~Hz}$ frequency with a $29 \operatorname{msec} \operatorname{period}(T \equiv 2 \pi / \omega)$.

\section{Viscous simple drop}

Using (72) and (74) for $n=2$ yields $\Delta \omega_{2} \cong 20 \mathrm{sec}^{-1}$ and $\omega_{2} \cong 200 \mathrm{sec}^{-1}$ $(T \cong 2 \pi / \omega \cong 31 \mathrm{msec})$. For $\mu_{i}=\mu_{s}=1(10)$ poise, $\Delta \omega_{2} \cong 24(25), \omega_{2} \cong 196$ (195) and $T_{2} \cong 32$ (32) msec; i.e., the damped frequency it is not very sensitive to $\mu_{s}$. Also, it is not much smaller than $\omega_{2}^{I}$. The damping time constant, from (75), is $\tau \cong 15,1.5,0.13 \mathrm{msec}$, for $\mu_{s}=0.1,1,10$ poise, respectively. The viscosity of the shell has a strong effect on thedamping rate (linear in $\mu_{s}$ for $\mu_{s} \gg \mu_{c}$ ). But perhaps the most important result is that the 
damping rate is quite strong i.e., the $29-32 \mathrm{msec}$ period of oscillation will be quickly damped out by viscosity. This is probably a very good result because it tells us that droplet oscillations-or droplet dynamics in general, most likely—will not have much effect on loss of sphericity.

\section{Inviscid compound drop}

Noting the rather small effect that viscosity had on the natural frequency of a simple drop permits us to more confidently use the inviscid compound drop results of Saffren et al., (1981). Thus, inserting our nominal droplet parameters into (71) yields the following $M_{i}^{(2)} \cong 1.30, M_{o}^{(2)} \cong 1.30, A_{2} \cong 1.34, \sqrt{B_{2}} \cong 1.06, C_{2} \cong 2.4 \times 10^{-5}$, giving $\omega_{+}^{(2)} \cong 368 \sec ^{-1}(59 \mathrm{~Hz})$ and $\omega_{-}^{(2)}=126 \sec ^{-1}(20 \mathrm{~Hz})$; i.e., the bubble mode's period is $\sim 17 \mathrm{msec}$ and that of the sloshing mode is $\sim 50 \mathrm{msec}$. For comparison, the "thin-shell" frequency, given by (68) with $\gamma=\gamma_{c}+\gamma_{s}=20$, gives $\omega_{2}^{I}=309 \mathrm{sec}^{-1}$, fairly close to the bubble mode (with no sloshing mode). If we had equivalent results as from Miller and Scriven (1968) for the simple drops, and we 'almost' do from Landman (1985), they would most likely tell us the 'same thing'; i.e., capillary waves are too ephemeral/evanescent to matter much. Don't worry that some jostling of the droplets will harm the MooR-unless perhaps it persists through the entire solidification process.

\section{E. Initial accelerations}

A simple (solid) drop with the equivalent density of $\bar{\rho}=1.0027$ when released from rest will have an initial acceleration given by $(90)$ as $\dot{u}_{o}=(1.0027-1.01) /(1.01+0.5) g=$ $-0.0048 \mathrm{~g}$; i.e., it will begin to fall with an initial acceleration of $\sim-4.74 \mathrm{~cm} / \mathrm{sec}^{2}$. At this rate, in one second its specd of $4.74 \mathrm{~cm} / \mathrm{scc}$ would far exceed the terminal velocity of $u_{T}=2 R^{2} g \Delta \rho / 9 \mu_{H} \cong 0.6 \mathrm{~cm} / \mathrm{sec}$. Thus, the 'response time' must be less than 1 second-as we shall soon verify.

The corresponding compound droplet's acceleration from rest, from (106) and (107), is $\dot{u}_{c}^{o} \cong-0.00089 \mathrm{~g}=-0.87 \mathrm{~cm} / \mathrm{sec}^{2}$ and $\dot{u}_{s}^{o} \cong-.0027 \mathrm{~g}=-2.64 \mathrm{~cm} / \mathrm{sec}^{2}$; the shell wants to fall three times faster than the core which, if surrounded fully by the 'shell' fluid, would actually want to rise, with an initial acceleration of $\dot{u}_{c}^{o}=\left(\rho_{s}-\rho_{c}\right) /\left(\rho_{c}+\frac{1}{2} \rho_{s}\right) g=0.0066 g$ $\cong 6.5 \mathrm{~cm} / \mathrm{sec}^{2}$. The free-stream (+ added mass) acceleration effect of the shell on the core causes the reversal which, as mentioned before, would be 'complete' in the thin-shell limit in that $\dot{u}_{c}^{0} \rightarrow \dot{u}_{s}^{o}$ for $\Delta R \rightarrow 0$. Of course this limit also calls into question the very meaning 
of the shell's equation of motion. But this limit, too, is actually well-behaved; (102) yields for this case-after setting $\dot{u}_{c}^{o}=\dot{u}_{s}^{o}$ there--the following reasonable result:

$$
\left(\rho_{c}+\frac{1}{2} \rho_{H}\right) \dot{u}_{s}^{o}=\left(\rho_{H}-\rho_{c}\right) g+\frac{3}{2} \rho_{H} \dot{u}_{H}^{o}
$$

which actually gives the core's initial acceleration, as required. (Note that now if $\rho_{c}=\rho_{H}$, this equation properly simplifies to $\dot{u}_{s}^{o}=\dot{u}_{H}^{o}$; the droplet simply follows the host fluid-an equation to which the core equation of motion also simplifies if $\rho_{c}=\rho_{s}=\rho_{H}$, in that it yields $\dot{u}_{c}^{o}=\dot{u}_{s}^{o}$.)

\section{F. Initial time constants.}

Finally, we shall employ (108)-(120) to provide estimates of the initial 'decay rate' of the above initial accelerations. We are, of course, seriously hampered by our lack of detailed knowledge of the viscous coupling coefficients, $K_{c s}, K_{c H}$, and $K_{s H}$, whose approximations we address next.

Assumption 1: Based on the small variation in the 'Hadamard-Rybczynski' drag

coefficient factor, $\frac{2 / 3+\mu_{c} / \mu_{s}}{1+\mu_{c} / \mu_{c}}$, with viscosity, we shall employ the equation that would be appropriate if the shell-core interface were rigid (an 'eggshell'), Eqn. (99), then reduce it by $25 \%$.

Assumption 2: We shall employ the steady-state relation of Brunn and Roden (1985), Eqn. (101) for $K_{c H}$.

Assumption 3: Here we shall use the steady-state result of Rushton and Davies (1983)_Eqn (103)_for $K_{s H}$. Thus, for the nominal case (with $\mu_{s}=1$ poise), we obtain $K_{c s} \cong 440, K_{c H} \cong .030$, and $K_{s H} \cong 0.996$. For the two extremes of shell viscosity $(0.1,10$ poise $)$, these become $K_{c s} \cong$ virtually no change, $K_{c H} \cong(0.33,0.0030)$, and $K_{s H} \cong(0.960,0.999)$. The small value of $K_{c H}$ means that the highly viscous shell has virtually decoupled the core from the host and the fact that $K_{s H} \cong 1$ tells us that the shell behaves more like a solid in the host fluid-again because it is very viscous, and thin.

We will use $K_{c s}=330, K_{c H}=0.03$, and $K_{s H}=1$ in our calculations. Thus, returning to $(108 \mathrm{ff})$, 


$$
\begin{aligned}
& k_{s H}=6 \pi \times .01 \times .109 \times 1 \cong 0.0205 \mathrm{gm} / \mathrm{sec} \\
& k_{c H}=6 \pi \times .01 \times .098 \times .03 \cong .0005 \\
& k_{c s}=6 \pi \times 1.0 \times .098 \times 330 \times 1 \cong 610 \\
& k_{s}=k_{c s}+k_{s H} \cong 610 \\
& k_{c}=k_{c s}+k_{c H} \cong 610 \\
& m_{c s}=\frac{4 \pi}{3}(.098)^{3} \times 1 \cong 0.00394 \mathrm{gm} \\
& m_{s c}=2 \pi(.098)^{3} \times 1.01 \times \frac{1}{1-(.9)^{3}}=0.0220 \\
& m_{s}=\frac{4 \pi}{3}\left\{\left[(.109)^{3}-(.098)^{3}\right] \times 1.01+0.5 \times(.109)^{3} \times 1\right\}=0.00421 \\
& m_{c}=\frac{4 \pi}{3}(.098)^{3}\left[1+1.01 \times \frac{(.9)^{3}+1 / 2}{1-(.9)^{3}}\right]=0.0220 \\
& c=k_{c} k_{s}-k_{c s}=k_{c s}\left(k_{c H}+k_{s H}\right)+k_{c H} k_{s H} \cong 12.8 \\
& b=-\left[m_{c} k_{s}+m_{s} k_{c}+\left(m_{c s}-m_{s c}\right) k_{c s}\right]=-4.9715 \\
& a=m_{c} m_{s}+m_{s c} m_{c s}=.000179 \\
& \therefore \lambda \pm=(4.9715 \pm 4.9705) / 2 \times .000179, \text { giving } \\
& \tau_{ \pm}=36 \mu \mathrm{sec}, 0.39 \mathrm{sec},
\end{aligned}
$$

and we note that the asymptotic estimates, given by (123) and (124), give virtually the same results and are thus quite accurate- because $k_{c s}$ totally dominates the friction terms. Thus, the core and shell will 'equilibrate' very quickly and then follow the host on a much slower time scale. Another way to say it is this: the encapsulated droplet behaves just about like a simple droplet - the core follows the shell, as usually scen in the laboratory. The 'effective' equation of motion for the compound drop-at least for $t>O\left(1 / \lambda_{+}\right)$-can thus probably be greatly simplified to

$$
\begin{aligned}
\left(m_{c}+m_{s}\right) \dot{u}= & \frac{4}{3} \pi\left[R_{s}^{3} \rho_{H}-R_{c}^{3} \rho_{c}-\left(R_{s}^{3}-R_{c}^{3}\right) \rho_{s}\right] g+\left(m_{s c}-m_{c s}\right) \dot{u}- \\
& 6 \pi \mu_{H} R_{s}\left[u-u_{H}+R \int_{0}^{1} \frac{\dot{u}(\tau)-\dot{u}_{H}(\tau)}{\sqrt{\pi(t-\tau)}} d \tau\right] .
\end{aligned}
$$

This equation gives an initial acceleration of

$$
\dot{u}(0)=\frac{\frac{4}{3} \pi\left[1.109^{3}-.098^{3}-1.01\left(.109^{3}-.093^{3}\right)\right] g}{m_{c}+m_{s}+m_{c s}-m_{c c}=.00815} \cong-0.0018 g \cong-1.8 \mathrm{~cm} / \mathrm{sec}^{2},
$$

which is in the same range as previously estimated.

Similarly, the initial time constant [where now "initial" must be properly interpreted: it is just after the very fast transient is completed, $t=O\left(1 / \lambda_{+}\right) \cong 36 \mu \mathrm{sec}$ ] can be approximated as 


$$
\tau_{-}=\frac{m_{c}+m_{s}+m_{c s}-m_{s c}}{6 \pi \mu_{H} R_{s}} \cong 0.40 \mathrm{sec},
$$

again agreeing closely with the more precise theory - and we conclude by stating that the compound drop will act more like a simple drop-or even like a solid drop-in that it will follow the host fluid with a time constant of about 0.4 seconds.

Finally, we believe that, while not explicitly proving that core centering must occur, the above analysis strongly suggests that the large viscous friction between core and (thin) shell will at least tend to keep the shell near the center.

\section{Conclusions and Future Work}

\section{A. Conclusions}

Based on what we have learned thus far, we offer the following conclusions:

1. It should be possible to get up a density column that does not cause a serious loss of sphericity of a stagnant compound drop. (The concomitant problem of much reduced mass transfer rate of shell solvent to aqueous host might cause excessive 'drying' times, however.)

2. The terminal velocity of a $2 \mathrm{~mm}$ drop with $\Delta \rho=0.01$ will be $\sim 0.3-0.7 \mathrm{~cm} / \mathrm{sec}$.

3. Droplets at terminal velocity will be close to violating the sphericity requirements.

4. Capillary waves at the two shell interfaces will oscillate in the lowest $\left(P_{2}\right)$ mode at $\sim 30-60 \mathrm{~Hz}$, but will be rapidly damped by viscosity.

5. Initial accelerations in the 'free-fall' mode will be in the range of $1-5 \mathrm{~cm} / \mathrm{sec}^{2}$

6. The transient response of the compound droplet shows two modes: a very fast (microsecond-millisecond range) 'equilibration' of core and shell and a much slower response $(\sim 0.4 \mathrm{sec})$ of the pair acting as a single droplet. The core wants to 'follow' the shell.

7. Thin shell hydrodynamics will usually be well-described by the transient Stokes equations because the internal flows will usually be very feeble. 


\section{B. Future Work}

Recommendations for follow-on efforts are listed below, although we are not yet sure of their 'priority'.

1. The design and construction of a 'shaky drop tower' might be worthwhile. The idea here is to shake the tower early in time while the 'fresh' droplets are beginning their free'fall (or rise, depending on densities) to keep the core centered while $\Delta \rho$ is at its largest value [part of the 'design'; $\Delta \rho$ should be largest initially and decreasing with time so that the droplet slows down as diffusion of organic solvent proceeds-giving a low terminal velocity (lower Re) later in time when sphericity is most important.] The oscillation amplitude would also be a decreasing function of time. This 'design' takes advantage of the large Schmidt numbers $(v / D$ where $D$ is the mass diffusivity) associated with mass transfer; i.e., we can simultaneously have a low Reynolds number $\left[O(1)\right.$ or less] and a high Peclet number $\left(P e \equiv u_{c} R / D\right)$ which enhances convective mass transfer. The "shaking" may be either physical or acoustic - and the drop tower might even be designed as a 'closed loop' with forced circulation of host fluid in order to keep the droplets in a narrow vertical zone.

2. Another mechanism for core centering that might be worthwhile analysing is the potential flow/acceleration effects of the shell on the core when the core is off-center. This would require the use of bipolar coordinates and would preclude simple ('hand') calculation—but should be easily do-able via computer.

3. Ditto 2 except examine, more quantitatively, the off-center viscous force on the core via the transient Stokes equations in bi-polar coordinates.

4. Investigate the possibility of acoustic oscillations to promote core centering.

5. Investigate the non-Newtonian fluid dynamics that surely must be present in the liquid shell-as it may seriously modify or even vitiate some or all of the 'Newtonian' conclusions reached thus far. (The shell probably behaves more viscoelastically than as a simple, constant-viscosity Newtonian fluid). The theoretical investigation (and selection of an appropriate non-Newtonian model for the constitutive equation) should be supplemented (if not preceded) by laboratory measurements of the shell fluids' rheology-including 'later time' behavior when the liquid becomes a 'solid', passing first through a 'gel' state. 


\section{Acknowledgements}

I am indebted to the following people, who helped me over one or more 'rough spots' during the research associated with this paper: J. Brady, P. Concus, P. Oguz, W-K. Rhim, R. Sani, H. Stone, and T. Wang. Finally, a special thanks to R. Cook, who introduced me to the entire subject of encapsulation and laser target fabrication - and who contributed materially and significantly to this paper. This work was performed under the auspices of the U.S. Department of Energy by Lawrence Livermore National Laboratory under contract number W-7405-Eng48.

\section{References}

Barthés-Biesel, D. and A. Acrivos. Deformation and Burst of a Liquid Droplet Freely Suspended in a Linear Shear Field. J. Fluid Mech., 61, 1973.

Basaran, O.A., T.C. Scott and C.H. Byers. Drop Oscillations in Liquid-Liquid Systems. AIChE Journal, 35, 1989.

Basset, A. On the Descent of a Sphere in a Viscous Liquid. Quarterly Journal of Pure and Applied Math, XLI, 1910 (p. 369).

Batchelor, G.K. An Introduction to Fluid Dynamics. Cambridge University Press, London, England, UK, 1967.

Brignell, A.S., The deformation of a liquid drop as small Reynolds number. Quarter. J. Mech., XXVI, 1973 (p. 99).

Brunn, P.O. and T. Roden. On the Deformation and Drag of a Type-A Multiple Drop at Low Reynolds Number. J. Fluid Mech., 160, 1985.

Chandrasekhar, S. Hydrodynamic and Hydromagnetic Stability. Oxford at the Clarendon Press. 1961.

Chisnell, R.F. The Unsteady Motion of a Drop Moving Vertically Under Gravity. J. Fluid Mech., 176, 1987.

Clift, R., J.R. Grace and M.E. Weber. Bubbles, Drops, and Particles. Academic Press, Inc., 1978. 
Cook, R., R. McEachern, and R. B. Stephens, Fusion Technol. (1998), this issue.

Czechowicz, D. and R. B. Stephens, Fusion Technol. (1998), this issue.

Dittrich, T. R., et al., Fusion Technol. 31, 402 (1997).

Gresho, P.M. and R.L. Sani, Incompressible Flow and the Finite Element Method: Advection-Diffusion and Isothermal Laminar Flow. John Wiley and Sons, 1998.

Gresho, P.M., A. Hindmarsh, and E. Michaelides. in preparation. 1998.

Haan, S. W., et al., Phys. Plasmas 2, 2480 (1995).

Hamilton, K.E., S.R. Buckley and R.R. Cook. The Science of NIF Scale Capsule Development. LLNL, UCRL-D-129331, 1997.

Happel, J. and H. Brenner. Low Reynolds Number Hydrodynamics with Special Applications to Particulate Media. Prentice-Hall Inc., Engelwood Cliffs, NJ, 1965.

Hjelmfelt, A.T., Jr., and L.T. Mockros. Stokes Flow Behavior of an Accelerating Sphere. Journal of the Engineering Mechanics Division, ASCE, 1967.

Kendall, J.M. Experiments on Annular Liquid Jet Instability and the Formation of Liquid Shells. Phys. Fluids, 29, 1986.

Kim, S. and S.J. Karrila. Microhydrodynamics: Principles and Selected Applications. Butterworth-Heinemann, 1991.

Koh, C.J. and L.G. Leal. The Stability of Drop Shapes for Translation at Zero Reynolds number Through a Quiescent Fluid. Phys. Fluids A, 1, 1989.

Lamb, Sir H. Hydrodynamics, Dover, 1945.

Landau, L.D. and E.M. Lifshitz. Fluid Mechanics. Pergamon Press, 1959.

Landman, K.A. Stability of a Viscous Compound Fluid Drop. AIChE Journal, 31, 1985.

Lawrence, C.J. and S. Weinbaum. The Force on an Axisymmetric Body in Linearized, Time-Dependent Motion: A New Memory Term. J. Fluid Mech., 171, 1986. 
Leal, L.G. Laminar Flow and Convective Transport Processes: Scaling Principles and Asymptotic Analysis. Butterworth-Heinemann, 1992.

Lee, C.P. and T.G. Wang. The Centring Dynamics of a Thin Liquid Shell in Capillary Oscillations. J. Fluid Mech., 188, 1988.

Lee, C.P., A.V. Anikumar and T.G. Wang. A Theoretical Model for Centering of a Thin Viscous Liquid Shell in Free and Forced Capillary Oscillations. Phys. Fluids, 8, 1996.

Lee, M.C., I-an Feng, D.D. Elleman, T.G. Wang and A.T. Young. Generation of a Strong Core-Centering Force in a Submillimeter Compound Droplet System. Proc. 2nd Int. Colloq. on Drops and Bubbles, D Le Croissette (Ed), JPL Publ. 82-7, 7, (1982).

Letts, S.A., M. Saculla, E. Fearon, S. Buckley, and R. Cook, Fusion Technol. (1998), this issue.

Levich, V.G. Physicochemical Hydrodynamics. Prentice-Hall, Inc., Englewood Cliffs, NJ, 1962.

Lovalenti, P.M. and J.F. Brady. The Force on a Bubble, Drop, or Particle in Arbitrary Time-Dependent Motion at Small Reynolds Number. Phys. Fluids A, 51993.

Maxey, M.R. and J.J. Riley. Equation of Motion for a Small Rigid Sphere in a Nonuniform Flow. Phys. Fluids, 26, 1983.

Maxey, M.R. The Equation of Motion for a Small Rigid Sphere in a Nonuniform or Unsteady Flow, p. 57 of Proceedings, Gas-Solid Flows, ASME FED-Vol 166 (1993).

Michaelides, E.E. Review-The Transient Equation of Motion for Particles, Bubbles, and Droplets. Journal of Fluids Engineering, ASME, 119, 1997.

Miller, C.A. and L.E. Scriven. The Oscillations of a Fluid Droplet Immersed in Another Fluid. J. Fluid Mech., 32, 1968.

Mockros, L.F., M. ASCE and R.Y.S. Lai. Validity of Stokes Theory for Accelerating. Spheres. Journal of the Engineering Mechanics Division, ASCE, 1969. 
Proudman, I. and J.R.A. Pearson. J. Fluid Mech., 2, 237, 1957.

Rushton, E. and G.A. Davies. Settling of Encapsulated Droplets at Low Reynolds Numbers. Int. J. Multiphase Flow, 9, 1983.

Sadhal, S.S. and H.N. Oguz. Stokes Flow Past Compound Multiphase Drops: The Case of Completely Engulfed Drops/Bubbles. J. Fluid Mech., 160, 1985.

Saffren, M., D.D. Elleman and W.K. Rhim. Normal Modes of a Compound Drop, Proc. Int. Colloq. on Drops and Bubbles, D. Le Coissette (Ed), JPL Pub. 87-7, 7 (1982).

Sy, F. and E.N. Lightfoot. Transient Creeping Flow Around Fluid Spheres. AIChE Journal, 17, 1971.

Taylor, T.D. and A. Acrivos. On the Deformation and Drag of a Falling Viscous Drop at Low Reynolds Number. J. Fluid Mech., 18, 1964.

Wang, T.G., A.V. Anilkumar, C.P. Lee, and K.C. Lin, Core-centering of compound drops in capillary oscillations: observations on USML-l experiments in space, J. Colloid and Interface Sci., 165, p.19 (1994).

Yang, S.-M. and L.G. Leal. A note on memory-integral contributions to the force on an accelerating spherical drop at low Reynolds number, Phys. Fl., A, 3 (7), July 1991. 


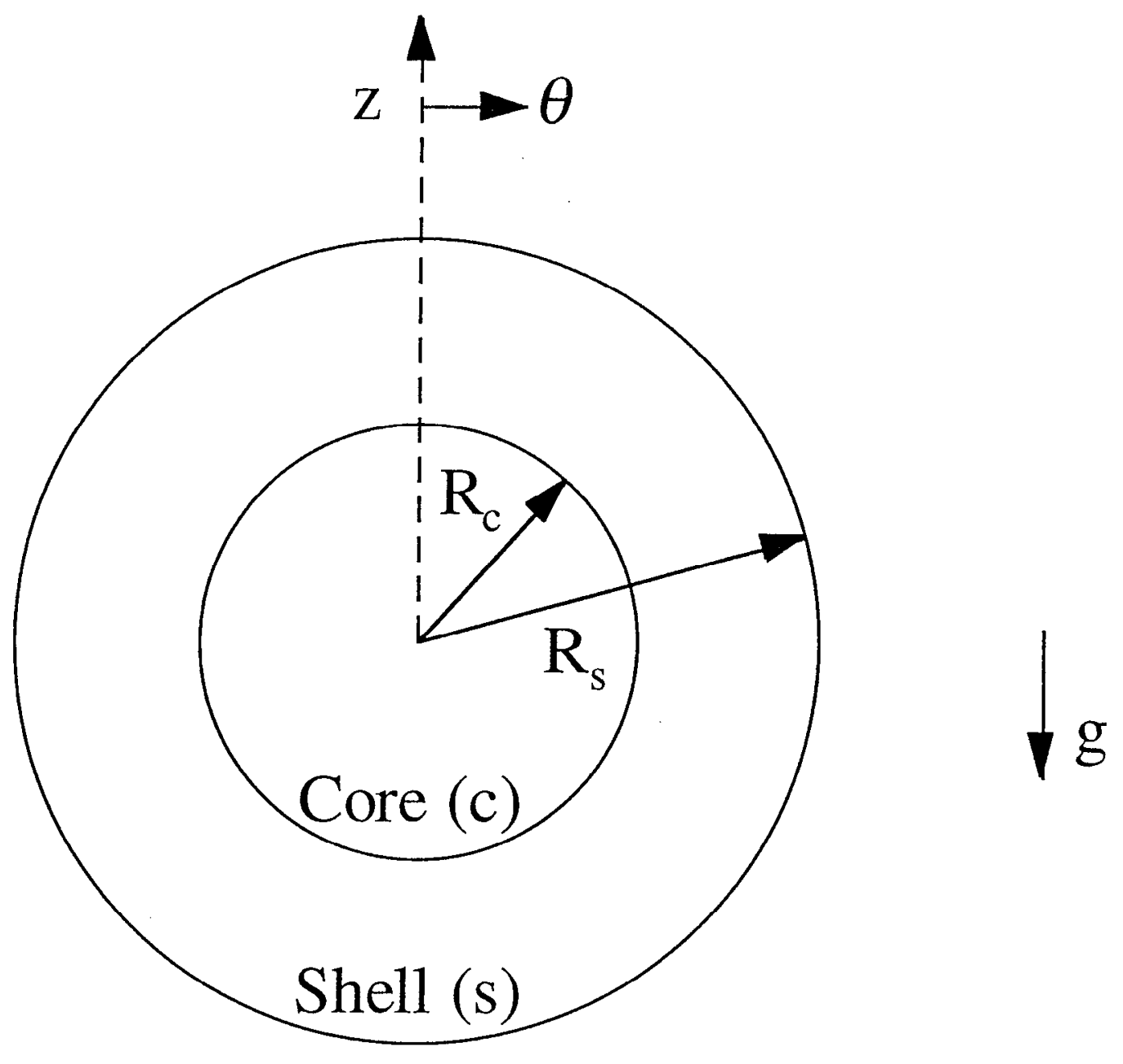

Host $(\mathrm{H})$

Fig. 1 Compound drop. 


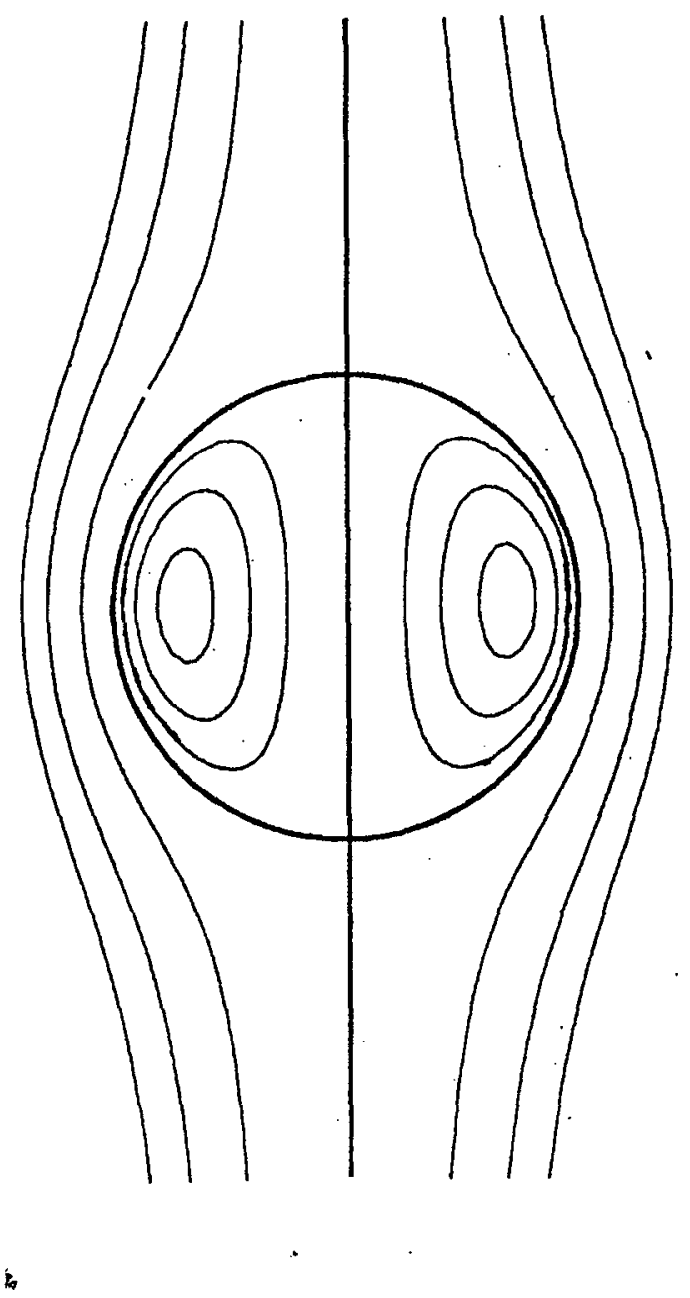

Fig. 2 Simple Drop; Steady Streamlines $\left(\mu_{i}=\mu_{o}\right)$. 


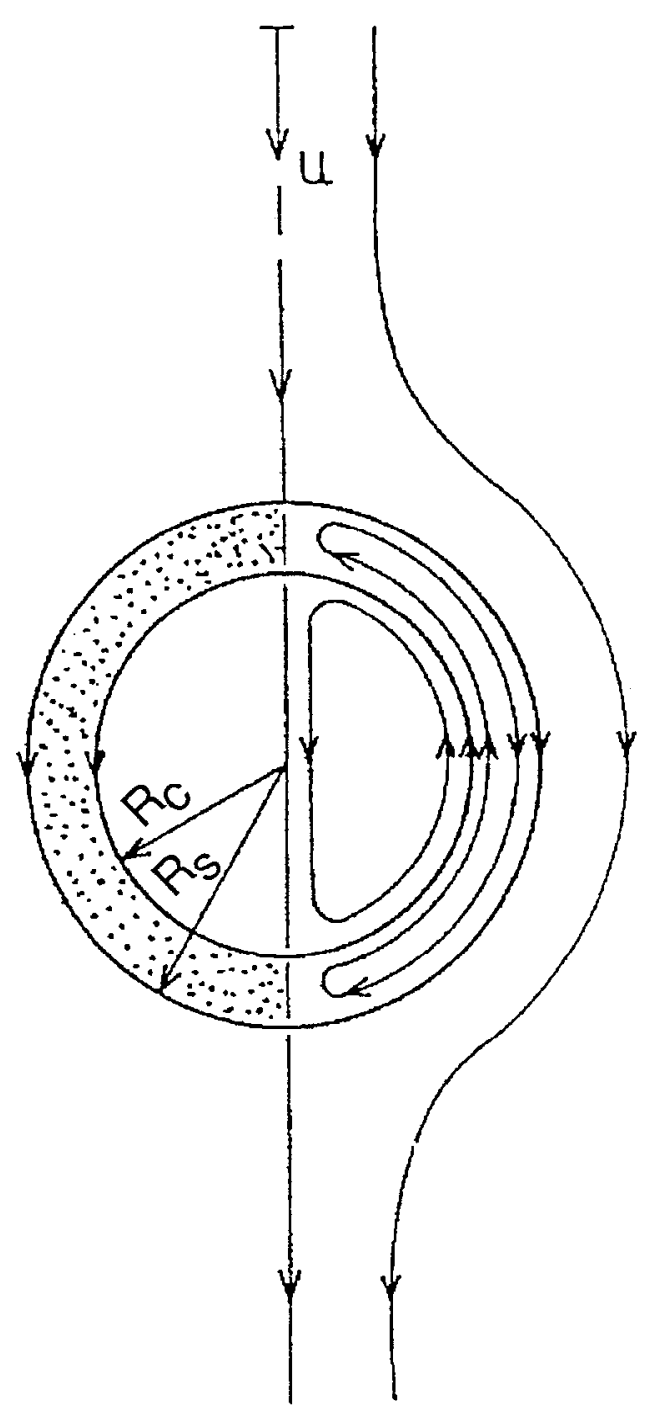

Fig. 3 Streamlines for a Compound Droplet. 

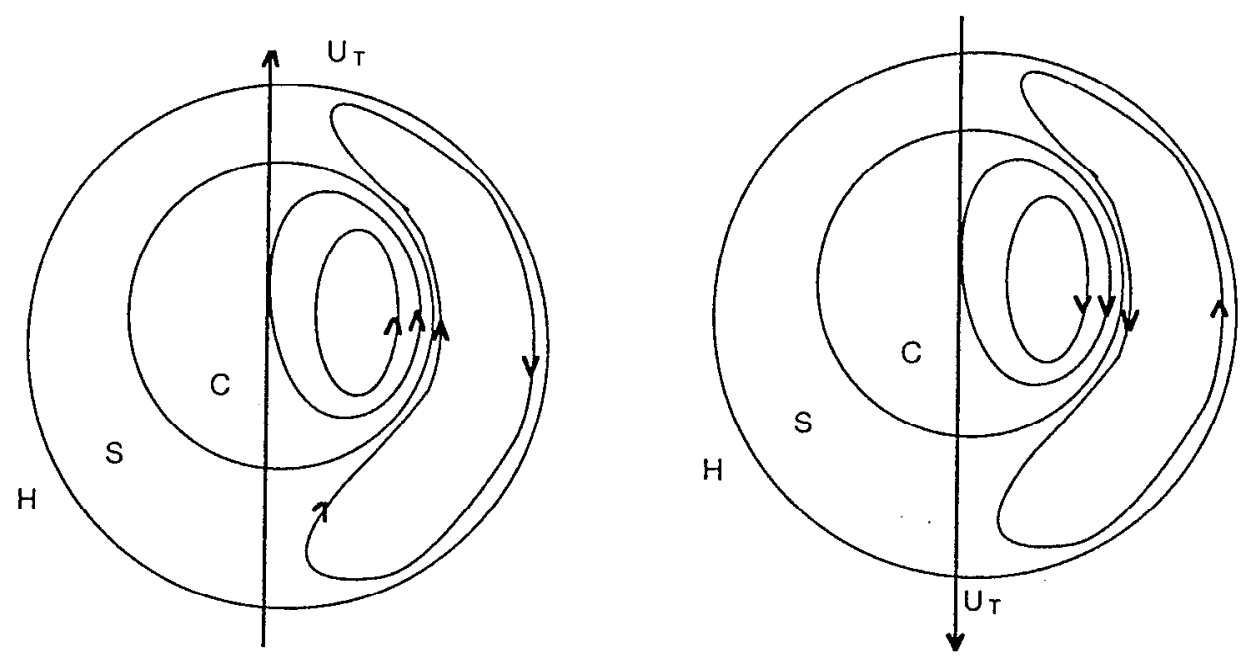

Fig. 4 Rising/Falling Compound Droplets- at $U_{T}$. 

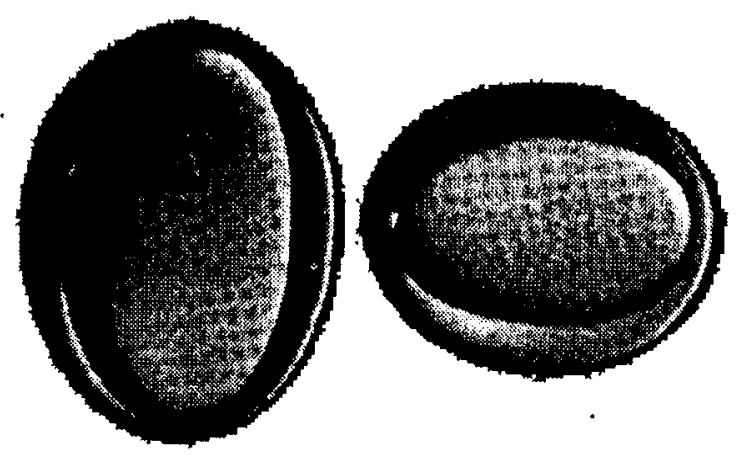

\section{Bubble Mode}

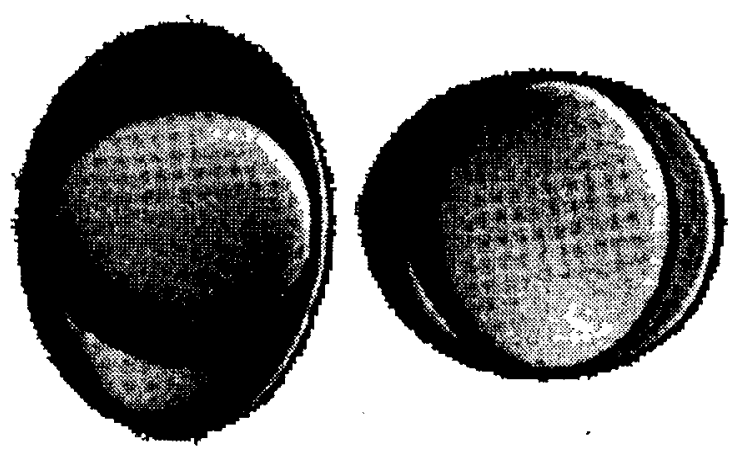

\section{Sloshing Mode}

Fig. 5 First two modes for a compound drop. 


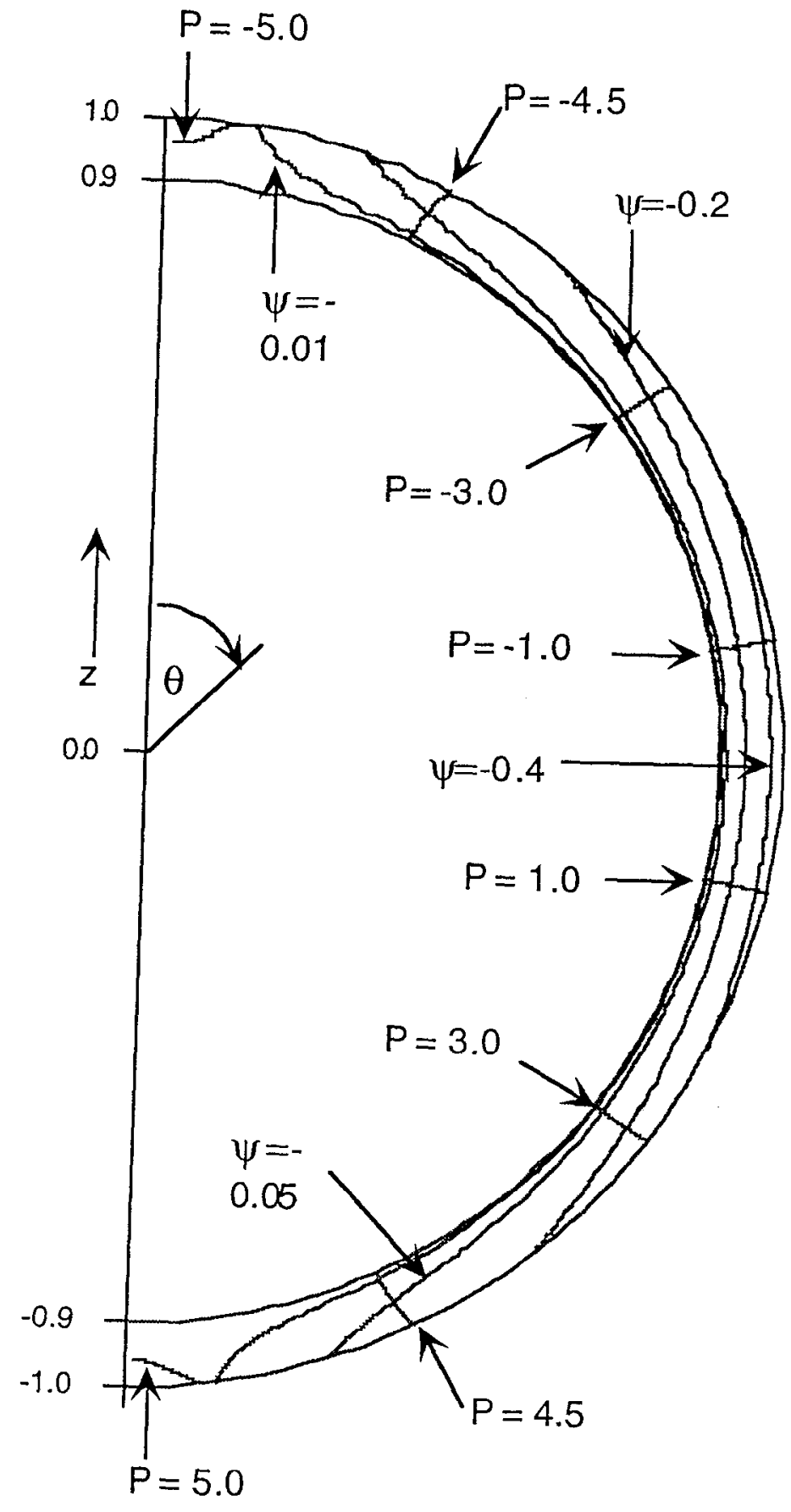

Fig. 6 Streamlines and Isobars for an (upward) accelerating shell. 


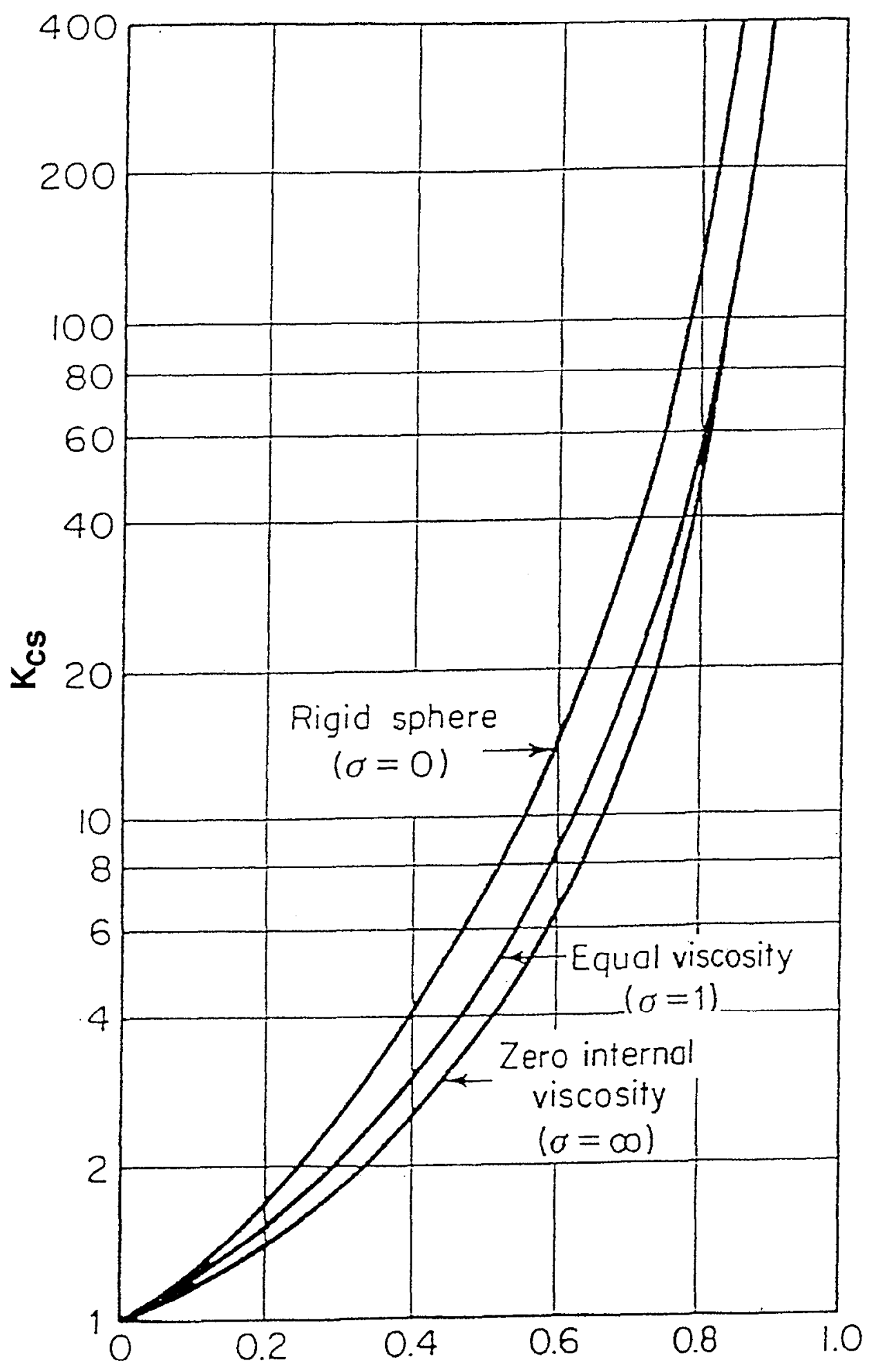

Fig. 7 Stokes' Law Correction Factor. 